\title{
Discovery of eclogite facies carbonate rocks from the Lindås Nappe, Caledonides, Western Norway
}

\author{
T. M. BOUNDY ${ }^{1}$, C. L. DONOHUE ${ }^{2}$, E. J. ESSENE ${ }^{2}$, K. MEZGER ${ }^{3}$ AND H. AUSTRHEIM ${ }^{4}$ \\ ${ }^{1}$ Department of Geosciences, University of Wisconsin-Milwaukee, Milwaukee, WI 53201, USA; \\ (tboundy@uwm.edu) \\ ${ }^{2}$ Department of Geological Sciences, University of Michigan, Ann Arbor, Michigan, 48109-1063, USA \\ ${ }^{3}$ Institut für Mineralogie, Universität Münster, Correnstrasse 24, Münster, Germany \\ ${ }^{4}$ Mineralogical-Geological Museum, Sarsgate 1, N-0562 Oslo, Norway
}

\begin{abstract}
Eclogite facies carbonate rocks have been discovered associated with the granulite-eclogite transitional rocks within Bergen Arc system, Caledonian Orogen of western Norway. The local occurrences of marbles and calc-silicates are found subparallel to the mafic eclogite facies shear zones on Holsnøy Island. Marbles contain the assemblage calcite $\left(\mathrm{Ca}_{0.99} \mathrm{Sr}_{0.01} \mathrm{CO}_{3}\right)$, calcian strontianite $\left(\mathrm{Ca}_{0.18-0.44} \mathrm{Sr}_{0.53-0.84} \mathrm{CO}_{3}\right)$, clinopyroxene $\left(\mathrm{Jd}_{7-32}\right)$, epidote/allanite $\left(\mathrm{Ps}_{0-33}\right)$, titanite, garnet $\left(\mathrm{Alm}_{52-56} \mathrm{Grs}_{28-33} \mathrm{Pyp}_{11-16}\right)$, barite $\left(\mathrm{Ba}_{0.90-0.99} \mathrm{Sr}_{0.01-0.10} \mathrm{SO}_{4}\right)$, celestine $\left(\mathrm{Sr}_{0.67-0.98} \mathrm{Ba}_{0.01-0.23} \mathrm{Ca}_{0.01-0.11} \mathrm{SO}_{4}\right)$, and one apparently homogeneous grain of intermediate composition $\left(\mathrm{Ba}_{0.49} \mathrm{Ca}_{0.01} \mathrm{Sr}_{0.50} \mathrm{SO}_{4}\right)$. Adjacent eclogites have clinopyroxene with similar jadeite contents $\left(\mathrm{Jd}_{14-34}\right)$ and similar garnet $\left(\mathrm{Alm}_{51-60} \mathrm{Grs}_{26-36} \mathrm{Pyp}_{8-14}\right)$ compositions. The marbles have high contents of Sr (9500-11000 p.p.m) and Y (115-130 p.p.m). However, low concentrations of some key trace elements (110-160 p.p.m. Ba and $<5$ p.p.m. Nb) appear to indicate that the marble is not a metamorphosed carbonatite. The ${ }^{87} \mathrm{Sr} /{ }^{86} \mathrm{Sr}$ ratios range from 0.7051 to 0.7059 . Field and petrological relationships suggest that metasomatic reactions and fluids played a significant role in producing and/or modifying the marbles. The breakdown of scapolite in the granulite into carbonates and sulphates during eclogite facies metamorphism may have contributed to the metasomatic formation of the marbles along shear zones.

Fluids involved during subduction are an important catalyst for metamorphism and are recognized to have played a critical role in the localized transformation from granulite to eclogite in the Holsnøy Island area. Thermobarometry indicates $640-690{ }^{\circ} \mathrm{C}$ and $18-20 \mathrm{kbar}$ for adjacent eclogites and temperatures of $580-650{ }^{\circ} \mathrm{C}$ for the calc-silicates. The marble assemblages are consistent with fluid that is dominantly comprised of $\mathrm{H}_{2} \mathrm{O}\left(\mathrm{X}_{\mathrm{CO}_{2}}<0.03\right)$ under high-pressure conditions. Phase equilibria of the marbles constrain the $f \mathrm{O}_{2}$ of the fluids and imply oxidizing conditions of the deep crustal fluids. At present the source of the fluids remains unresolved. The results provide additional insights into the variable and evolving nature of fluids related to subduction and high-pressure metamorphism.
\end{abstract}

Key words: calcian strontianite; eclogite; fluids; marble; Norway.

\section{INTRODUCTION}

Marbles associated with regional occurrences of eclogites provide a unique perspective for evaluating metamorphic processes and fluids associated with crustal subduction. Such marbles have been described from the Tauern Window within the Austrian Alps (Franz \& Spear, 1983), from the Pan-African belt within Mali (Caby, 1994) and associated with coesitebearing eclogites from the Dabie Mountains in central China (e.g. Wang \& Liou, 1991; Schertl \& Okay, 1994; Zhang \& Liou, 1996). Siliceous dolomites from the Tauern window indicate an early assemblage of diopside, tremolite, dolomite, calcite, quartz and zoisite stable at fluid pressures of 18-25 kbar (Franz \& Spear, 1983). Impure marbles from the Dabie Mountains contain occasional coesite in dolomite (Schertl \&
Okay, 1994), coesite, quartz pseudomorphs after coesite, aragonite, and calcite pseudomorphs after aragonite occurring as fine-grained inclusions in garnet and omphacite (Wang \& Liou, 1993) indicating that both the marbles and associated eclogites experienced ultra-high pressure metamorphism ( $>27$ kbar). Evaluation of phase equilibria for both the marbles from the Tauern Window and the Dabie Mountain indicates fluid compositions with very low $X_{\mathrm{CO}_{2}}$ similar to those inferred in the associated eclogite facies rocks (e.g. Franz \& Spear, 1983; Holland, 1979; Wang \& Liou, 1993). The emerging picture of the fluid evolution during high-pressure metamorphism suggests their compositions can be both complex and highly variable (e.g. Yardley, 1997).

Marbles occur associated with the regionally exposed granulite to eclogite transitional rocks in the Lindås 
Nappe of the Bergen Arcs system (BAS), western Norway. The eclogites formed locally in the granulites as a result of deformation and the introduction of fluids (Austrheim \& Griffin, 1985; Austrheim, 1987, 1990). Calcite and dolomite occur as part of the equilibrium assemblage of a few eclogites (Jamtveit et al., 1990). Local outcrops of marbles are also associated with the eclogites. Marbles from the Liavatn area on Holsnøy Island (Fig. 1) have calcite with average $\delta^{18} \mathrm{O}$ of $9.5 \pm 0.6 \%$ and $\delta^{13} \mathrm{C}$ of $-4 \pm 0.8 \%$ that are in equilibrium with the silicate minerals (van Wyck et al., 1996). The data are interpreted to reflect retrogression due to the influx of fluids at temperatures of approximately $600{ }^{\circ} \mathrm{C}$. There is a considerable range in estimates of composition of the eclogite fluid phase. Based on phase equilibria in the eclogites (sensu stricto) from Holsnøy the fluid composition during eclogite facies metamorphism was estimated to have been $\mathrm{H}_{2} \mathrm{O}$-rich $\left(X_{\mathrm{H}_{2} \mathrm{O}}>0.75\right.$; Jamtveit et al., 1990). Fluid inclusions found in quartz within eclogite facies quartz veins contain abundant $\mathrm{N}_{2}$ together with $\mathrm{CO}_{2}$, whereas $\mathrm{H}_{2} \mathrm{O}$ was not detected (Andersen et al., 1990). Multiphase brine inclusions in omphacite are interpreted to reflect enrichment of elements incompatible with the silicate structure during consumption of $\mathrm{H}_{2} \mathrm{O}$ by eclogiteforming hydration reactions (Svensen et al., 1999, 2001), suggesting the infiltration of water-rich brines. There is no reason to assume the pervasive invasion of a single fluid. The carbonate rocks occur parallel to or along eclogite shear zones in the area. The composition of fluids in equilibrium with the carbonates may not have been the same as that in the mafic eclogite during eclogite facies metamorphism.

In this contribution, we present initial results from an investigation of carbonate rocks associated with the eclogite facies shear zones on Holsnøy Island within the Lindås Nappe of the BAS. The mineralogy of the carbonates includes unusual high-Sr minerals including calcian strontianite. We document field and petrological relations of the high-pressure marbles and calc-silicates in tandem with phase equilibria and $\mathrm{Sr}$ isotope characteristics. The composition of clinopyroxene in the marbles and the field association demonstrate that the marbles were also subjected to eclogite facies conditions. Investigation into the phase equilibria yields information not only of the $P-T$ conditions but also the constraints on the variable fluid compositions in equilibrium with the eclogite facies rocks. The results provide insights into the possible fluid mediated metasomatic formation of the marbles concurrent with eclogite facies metamorphism during deep subduction of the continental crust.

\section{GEOLOGICAL AND TECTONIC FRAMEWORK}

The development of the mid-Palaeozoic Scandinavian Caledonian Orogen (SCO) is related to the convergence of Laurentia (Greenland) and Baltica (Scandinavia). The SCO is characterized by a series of thrust nappes generally displaced from west to east over the Baltic Shield during the main phase of continentcontinent collision (Roberts \& Gee, 1985). Extensive erosion and late to postorogenic extension led to the present tectonic juxtaposition of lowermost crustal and upper crustal rocks (e.g. Andersen \& Jamtveit, 1990). Within the SCO of western Norway eclogite facies rocks occur locally in the Western Gneiss Region (WGR) and within the overlying Caledonian Nappes of the Bergen Arcs system (BAS). One of the arcuate Caledonian nappes of the Bergen Arcs, the Lindås Nappe, consists of a granulite facies anorthosite complex and is interpreted to represent a slice of the lowermost continental crust now exposed over $c .1000 \mathrm{~km}^{2}$ (Austrheim \& Griffin, 1985).

The Lindås Nappe anorthosite complex records two main phases of deformation and metamorphism. Late

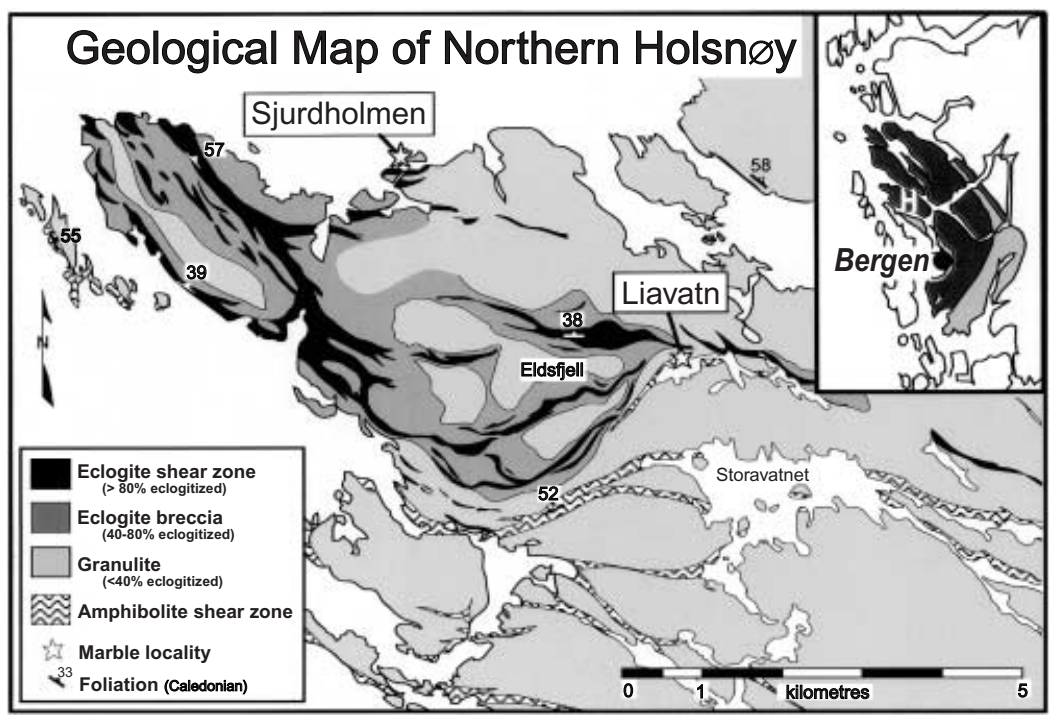

Fig. 1. Geological map of the NW Holsnøy Island area, Bergen Arcs, western Norway. Eclogites occur locally within the granulites as diffuse patches and along fractures and shear zones. The major eclogite facies shear zones illustrated on the map form an anastomosing network within the partially eclogitized granulites. Marble and related eclogite samples for this study are from the Liavatn and Sjurdholmen localities. 
Proterozoic (Sveconorwegian) garnet-granulite facies metamorphism and deformation occurred at deep crustal levels followed by a second period of deformation and metamorphism during the Caledonian Orogeny that brought the granulites to eclogite facies conditions (Austrheim \& Griffin, 1985). However, not all the granulite converted to eclogite. The localized transition from granulite to eclogite facies rocks took place over a few centimetres apparently as a result of deformation and the infiltration of fluids. The eclogites are best preserved on Holsnøy Island, in the northwestern part of the Lindås Nappe. There the eclogites formed along fractures within the granulites, along shear zones up to $120 \mathrm{~m}$ thick and continuous for several kilometres, and in breccias where angular blocks of granulite are surrounded by anastomosing, well-foliated eclogite (Austrheim, 1987; Klaper, 1991; Boundy et al., 1992). In addition, significant volumes of the partially eclogitized granulites contain eclogites that are apparently unrelated to any high strain zone. Such granulites contain isolated patches of eclogite and are presumably related to the infiltration of fluids along microfractures (Fig. 1). In addition to the map units, several different eclogite occurrences have recently been recognized, though at a scale that prevents their inclusion as map units. Austrheim \& Boundy (1994) documented eclogitic pseudotachylytes related to the eclogite facies metamorphism. Marbles and calc-silicates are associated with the eclogites in a few isolated areas (Boundy, 1995; Boundy et al., 1997a).

\section{FIELD RELATIONS OF THE MARBLES}

Marbles occur associated with the eclogites and eclogite facies shear zones on Holsnøy Island. The area consists of granulites, partially eclogitized granulites and eclogites (Fig. 1). On the map scale these eclogites are depicted as major eclogite facies shear zones. Liavatn and Sjurdholmen are the two most extensive marble localities discovered thus far (Fig. 1). The marble locality at Liavatn was mapped in detail (1:5000; Fig. 2). Unfortunately, the marbles are not well exposed, and thus the mapping depicts their minimum extent. At the Liavatn locality, marbles occur in a zone that parallels an eclogite facies shear zone, which is variably retrograded to amphibolite facies (Fig. 2). The eclogite shear zone and subparallel marble bodies occur within a large body of granulite facies anorthosites and are associated locally with high Fe-Ti ultramafic lenses. The zone of marbles is approximately $400 \mathrm{~m}$ long and up to $50 \mathrm{~m}$ thick, although the thickness is quite variable. Despite the poor exposure, it appears that the marble zone is severely attenuated towards the west (Fig. 2). The northern boundary of the marbles is formed by an eclogite zone that lies structurally above the marbles. Locally marble is intercalated with the eclogite shear zone along layers parallel to the foliation (Fig. 3). Retrogressed anorthosite shear zone rocks, termed 'paper schist' due to the extremely thin foliation layers, occur along the southern boundary, structurally below the marbles, and also occur locally along the northern boundary. The retrograde shear zones are amphibolite and lower grade (Fig. 2).

The marbles at the Liavatn locality are strongly deformed. At the macroscopic level, the foliation is defined by epidote/pyroxene rich layers within the carbonate matrix (Fig. 3). Generally this foliation is subparallel to the bounding eclogite and retrograde shear zones. In several areas the foliation is folded. Lenses of eclogite occur locally within the marbles and are typically $5-30 \mathrm{~cm}$ in diameter. Other mafic layers within the carbonates are comprised of mainly biotite

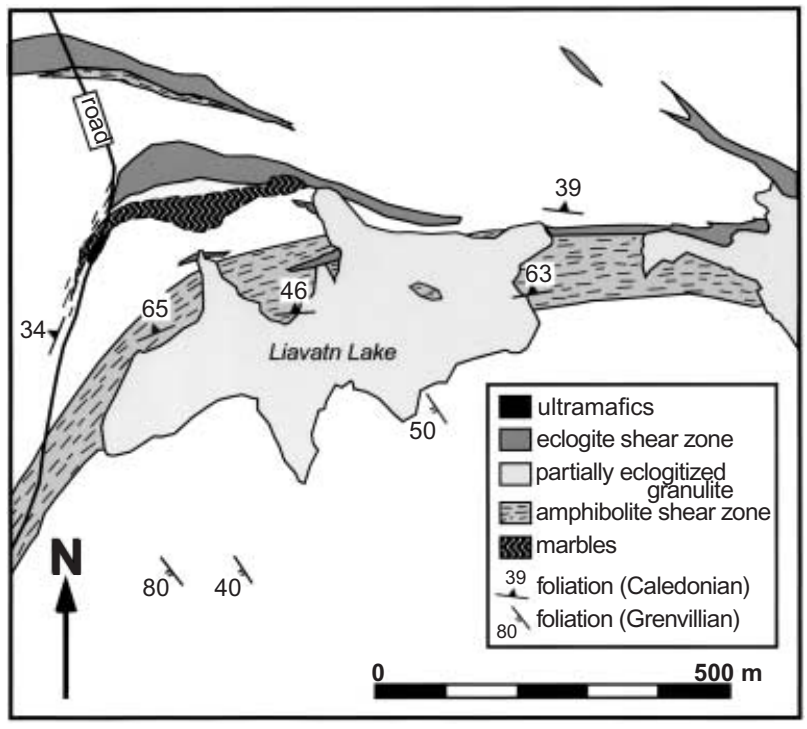

Fig. 2. Detailed map of the Liavatn marble locality on NW Holsnøy Island area. Locally the marble includes layers and boudins of eclogite and anorthosite 'paper schist'.

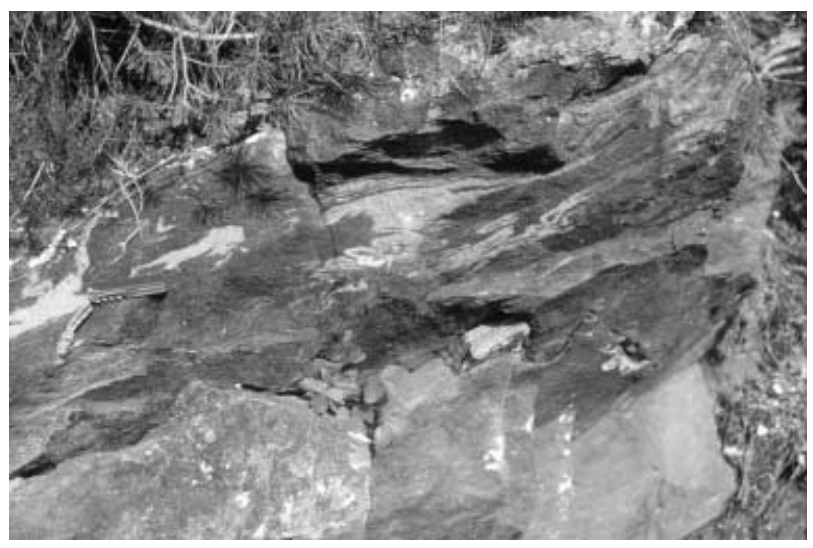

Fig. 3. Field photograph of marble interlayered with eclogite at the Liavatn locality. The foliation of the marble is defined by epidote and pyroxene-rich layers and parallels that in the adjacent well-foliated eclogite. Part of a Swiss army knife is shown for scale (left edge of photo).

and titanite and may represent retrograded eclogite lenses. Some of the eclogite layers adjacent to the marbles contain veins of quartz, omphacite, clinozoisite and calcite. The veins are discontinuous and are typically on the order of $1 \mathrm{~cm}$ thick and $10 \mathrm{~cm}$ long. One eclogite sample at Liavatn displays cavities with carbonates and/or clinopyroxene crystals protruding into the cavity. The veins and cavities suggest that fluid pressure approximated load pressure in these rocks.

At Sjurdholmen, an island off the NW coast of Holsnøy, the marbles occur in layers from $<5 \mathrm{~cm}$ to $3 \mathrm{~m}$ thick (Fig. 1). Along the shoreline, these outcrops are well exposed. Thin carbonate bands are interlayered with the eclogites and retrogressed eclogites. The eclogites contain centimetre thick, discontinuous quartz-muscovite veins. Metre thick marble layers are parallel to the general foliation in the area. A quartz layer on the order of $50 \mathrm{~cm}$ thick lies structurally above the thick marble layers. Eclogites occur as boudins or lenses within the quartz layer. Retrogressed mangerites bound the package of eclogite, marble and quartz layer, both structurally above and below. 
The protolith to the eclogites in this area appears to be predominantly mangerite, which at this locality is heavily retrogressed (to amphibolite facies or lower grade). At the macroscopic scale, epidote/ pyroxene-rich layers define the foliation of these highly deformed marbles, similar to those at Liavatn. The foliation is generally subparallel to that in the adjacent layers, although some folding also occurs.

In many cases the protolith for the eclogites may be deduced from the field relationships, and are typically granulites of anorthositic to gabbroic composition, although jotunite, mangerite and locally ultramafic granulite protoliths have also been found (Austrheim \& Griffin, 1985; Austrheim, 1990). For most of the amphibolite and greenschist facies shear zones it is likewise the case that the protolith can be deduced from the relationships observed in the field. However, given the minor and localized occurrence of marbles studied, a protolith is not evident based solely on the field relationships. The Liavatn marble body is located entirely within a large anorthosite

Table 1. Description of the marble and adjacent eclogite samples.

\begin{tabular}{|c|c|c|c|c|}
\hline \multirow[b]{2}{*}{ Sample } & & \multirow[b]{2}{*}{ Mineral assemblage } & \multicolumn{2}{|c|}{ Temperature estimate } \\
\hline & & & cc-dol ${ }^{1}$ & grt-cpx ${ }^{2}$ \\
\hline \multicolumn{5}{|c|}{ Liavatn locality } \\
\hline $\mathrm{HA} 4 / 90 \mathrm{~A}$ & marble & $\mathrm{cc}, \mathrm{ep}, \mathrm{ttn}, \mathrm{ap}, \mathrm{cel}, \mathrm{ms}$ & $\geq 460{ }^{\circ} \mathrm{C}$ & \\
\hline HA5/90 & marble & $\mathrm{cc}, \mathrm{ep}, \mathrm{ttn}, \mathrm{ap}, \mathrm{cel}, \mathrm{ms}$ & $\geq 390{ }^{\circ} \mathrm{C}$ & \\
\hline BA9/91 A & marble & cc, ttn, ep, cel, bar (dol) & $\geq 490^{\circ} \mathrm{C}$ & \\
\hline BA9/91 A' & marble & $\mathrm{cc}, \mathrm{ttn}, \mathrm{omp}, \mathrm{ep}, \mathrm{cel}, \mathrm{bar}$ & $\geq 460{ }^{\circ} \mathrm{C}$ & \\
\hline BA9/92 A & calc-silicate & $\mathrm{cc}, \mathrm{ep}, \mathrm{ms}, \mathrm{ttn}$ & $\geq 450^{\circ} \mathrm{C}$ & \\
\hline BA9/92B & marble & $\mathrm{cc}, \mathrm{ep}, \mathrm{ttn}, \mathrm{ap}, \mathrm{bt}, \mathrm{ms}$ (chl) & - & \\
\hline BA21/93 & calc-silicate & $\begin{array}{l}\text { cc, ep, grt, ms, rut, } \\
\text { ap, omp (sympl) }\end{array}$ & $\geq 620{ }^{\circ} \mathrm{C}$ & $580-650{ }^{\circ} \mathrm{C}$ \\
\hline Liavatn & eclogite & omp, grt, ru, ttn, cc, ms & - & $640-690^{\circ} \mathrm{C}$ \\
\hline \multicolumn{5}{|c|}{ Sjurdholmen locality } \\
\hline Sample & & Mineral assemblage & & \\
\hline BA25/92 A & marble & $\begin{array}{l}\text { cc, cpx, all, ttn, ap, } \\
\text { cel, bar, bt, op (hbd) }\end{array}$ & $\geq 350{ }^{\circ} \mathrm{C}$ & \\
\hline BA25/92B & marble & $\begin{array}{l}\text { cc, cpx, all, ttn, ap, } \\
\text { bt, cel, op, ilm? (hbd) }\end{array}$ & $\geq 440{ }^{\circ} \mathrm{C}$ & \\
\hline BA69/93 & eclogite & $\begin{array}{l}\text { cpx, grt, all, cc, ttn, } \\
\text { ru, ap (sympl) }\end{array}$ & - & $595^{\circ} \mathrm{C}$ \\
\hline BA70/93 & calc-silicate & $\begin{array}{l}\text { cpx, cc, ttn, ap, grt, } \\
\text { qtz, alb, bar, zrc, ep }\end{array}$ & $\geq 280^{\circ} \mathrm{C}$ & \\
\hline
\end{tabular}

Abbreviations: all, allanite; ap, apatite; bar, barite; bt, biotite; cc, calcite; cel, celestine; chl, chlorite; cpx, clinopyroxene; dol, dolomite; epd, epidote; grt, garnet; hbd, hornblende; ilm, ilmenite; mu, muscovite; omp, omphacite; plg, plagioclase, op, opaque; qz, quartz; ru rutile; sympl, symplectite; ttn, titanite; zrc, zircon. () indicates retrogressive phase.

${ }^{1}$ Anovitz \& Essene (1987)

${ }^{2}$ Krogh Ravna (2000). body, comprised of anorthosites, gabbros and, locally, ultramafic rocks.

\section{DESCRIPTION OF ASSEMBLAGES}

Samples were collected from the two localities shown (Fig. 1); mineral assemblages are given in Table 1 and mineral analyses in Tables 2-10. The marbles have a fairly uniform grain size $(0.3-1.0 \mathrm{~mm})$. At both the Liavatn and Sjurdholmen localities the marbles consist predominantly of calcite along with minor epidote/allanite, titanite, apatite and celestine. Typically, calcite appears homogeneous with sharp grain boundaries. At the microscopic scale some calcite, however, has oriented inclusions of celestine (Fig. 4a). Clinopyroxene occurs in Liavatn marble samples BA9/91 A' (= BA10/92) and as inclusions in garnet porphyroblasts from sample BA21/93. Clinopyroxene shows symplectitic breakdown textures, in part to hornblende. Most of the clinopyroxene appears unaffected by this retrogression. Clinopyroxene in marbles from both the Liavatn and Sjurdholmen localities displays bright emerald green pleochroism. Garnet occurs in one of the calcsilicate samples from the Liavatn locality (BA21/93). The subhedral grains of garnet are full of inclusions, mainly carbonate with omphacitic clinopyroxene and epidote. The sample also includes areas of symplectite, which in part is hornblende, but no relict clinopyroxene was found outside of the garnet porphyroblasts in this sample. Epidote and allanite from the marbles show dramatic zonation (Fig. 4b). The eclogite samples from both localities consist of omphacite, garnet, rutile, titanite, calcite and quartz. The omphacite shows symplectitic breakdown textures mainly along the grain boundaries. Some mafic layers within the marbles consist of rutile with titanite overgrowths, garnet and symplectite with relict omphacite and are interpreted to represent a lens of retrograded eclogite within the marble.

\section{ANALYTICAL METHODS}

The minerals were studied by back-scattered electron (BSE) imaging and analysed (Tables 2-10) with a Cameca CAMEBAX electron microprobe using a wavelength dispersive system and a PAP correction routine at the University of Michigan. The BSE images were used as a guide to detect potential compositional zonation in the phases and to aid in determining the areas of interest for analysis. Some of the BSE images are shown in Fig. 4(a,b). All samples were analysed at an accelerating voltage of $15 \mathrm{kV}$ and a beam current of $10 \mathrm{nA}$ with the exception of some carbonates for which the beam current was reduced to $7 \mathrm{nA}$ to minimize elemental migration. The elements $\mathrm{F}, \mathrm{Na}, \mathrm{Cl}$ and $\mathrm{K}$ were measured at the beginning of each analysis to minimize errors associated with their migration. The $\mathrm{F}$ and $\mathrm{Cl}$ were measured for

Table 2. Carbonate analyses.

\begin{tabular}{|c|c|c|c|c|c|c|c|c|c|c|}
\hline & $\begin{array}{c}\mathrm{HA} 4 / 90 \mathrm{~A} \\
5 / 1\end{array}$ & $\begin{array}{c}\text { HA } 5 / 90 \\
4 / 1\end{array}$ & $\begin{array}{c}\text { BA9/91 A } \\
4 / 1\end{array}$ & $\begin{array}{c}\text { BA9/91 A } \\
1 / 1\end{array}$ & $\begin{array}{c}\text { BA } 9 / 92 \\
2 / 2\end{array}$ & $\begin{array}{c}\mathrm{BA} 21 / 93 \\
3 / 1\end{array}$ & $\begin{array}{r}\mathrm{BA} 21 / 93 \\
2 / 3^{*}\end{array}$ & $\begin{array}{c}\mathrm{BA} 25 / 92 \mathrm{~A} \\
1 / 2\end{array}$ & $\begin{array}{c}\mathrm{BA} 25 / 92 \mathrm{~B} \\
3 / 5\end{array}$ & $\begin{array}{c}\text { BA70/93 } \\
3 / 2\end{array}$ \\
\hline $\mathrm{FeO}$ & 1.55 & 1.07 & 1.73 & 1.60 & 1.25 & 3.11 & 3.44 & 1.10 & 1.75 & 0.72 \\
\hline $\mathrm{MnO}$ & 0.13 & 0.22 & 0.18 & 0.19 & 0.26 & 0.19 & 0.00 & 0.33 & 0.36 & 0.51 \\
\hline $\mathrm{SrO}$ & 0.65 & 1.14 & 0.80 & 0.69 & 0.00 & 0.00 & 1.21 & 0.00 & 0.24 & 0.00 \\
\hline $\mathrm{MgO}$ & 1.38 & 0.91 & 1.39 & 1.35 & 1.14 & 2.90 & 3.14 & 0.67 & 0.96 & 0.52 \\
\hline $\mathrm{CaO}$ & 50.64 & 51.42 & 50.65 & 51.06 & 52.85 & 50.04 & 46.30 & 53.67 & 51.63 & 53.29 \\
\hline $\mathrm{CO}_{2}$ & 45.65 & 45.24 & 45.65 & 45.11 & 43.65 & 44.45 & 45.91 & 43.72 & 42.96 & 43.14 \\
\hline $\mathrm{Mg}$ & 0.04 & 0.02 & 0.04 & 0.03 & 0.03 & 0.07 & 0.08 & 0.02 & 0.02 & 0.01 \\
\hline $\mathrm{Fe}$ & 0.00 & 0.00 & 0.02 & 0.00 & 0.02 & 0.04 & 0.05 & 0.02 & 0.02 & 0.01 \\
\hline $\mathrm{Mn}$ & 0.02 & 0.02 & 0.00 & 0.02 & 0.00 & 0.00 & 0.00 & 0.00 & 0.01 & 0.01 \\
\hline $\mathrm{Sr}$ & 0.01 & 0.01 & 0.01 & 0.01 & 0.00 & 0.00 & 0.01 & 0.00 & 0.00 & 0.00 \\
\hline $\mathrm{Ca}$ & 0.93 & 0.95 & 0.93 & 0.93 & 0.94 & 0.89 & 0.81 & 0.96 & 0.94 & 0.95 \\
\hline $\mathrm{T}\left({ }^{\circ} \mathrm{C}\right) \mathrm{cc}-\mathrm{dol} 1^{1}$ & 460 & 390 & 490 & 460 & 450 & 620 & - & 350 & 440 & 280 \\
\hline
\end{tabular}

${ }^{1}$ Anovitz \& Essene (1987)

$*$ included in garnet. 
Table 3. Orthorhombic carbonate analyses.

\begin{tabular}{|c|c|c|c|c|c|c|c|c|}
\hline Sample/Point & BA9/91 A 1 & BA9/91 A 2 & BA9/91 A 4 & BA9/91 A 5 & BA9/91 A 6 & BA9/91 A 7 & BA9/91 A 8 & BA9/91 A 9 \\
\hline $\mathrm{CaO}$ & 7.12 & 16.20 & 15.41 & 18.20 & 19.16 & 18.96 & 19.47 & 13.86 \\
\hline $\mathrm{SrO}$ & 61.58 & 48.99 & 50.16 & 45.29 & 47.15 & 44.42 & 45.89 & 52.29 \\
\hline $\mathrm{MgO}$ & 0.00 & 0.00 & 0.00 & 0.00 & 0.00 & 0.00 & 0.00 & 0.00 \\
\hline $\mathrm{BaO}$ & 0.05 & 0.09 & 0.03 & 0.00 & 0.00 & 0.00 & 0.00 & 0.00 \\
\hline $\mathrm{PbO}$ & 0.01 & 0.00 & 0.00 & 0.00 & 0.00 & 0.00 & 0.00 & 0.00 \\
\hline $\mathrm{FeO}$ & 0.12 & 0.51 & 0.08 & 0.01 & 0.03 & 0.01 & 0.06 & 0.06 \\
\hline $\mathrm{MnO}$ & 0.04 & 0.02 & 0.01 & 0.00 & 0.00 & 0.00 & 0.00 & 0.00 \\
\hline $\mathrm{CO}_{2}$ & 30.82 & 34.29 & 34.61 & 37.52 & 33.19 & 37.58 & 34.51 & 34.01 \\
\hline Total & 99.74 & 100.10 & 100.29 & 101.02 & 99.53 & 100.98 & 99.93 & 100.22 \\
\hline $\mathrm{Ca}$ & 0.18 & 0.37 & 0.36 & 0.40 & 0.44 & 0.42 & 0.44 & 0.32 \\
\hline $\mathrm{Sr}$ & 0.83 & 0.61 & 0.63 & 0.54 & 0.59 & 0.53 & 0.56 & 0.66 \\
\hline $\mathrm{Mg}$ & 0.00 & 0.00 & 0.00 & 0.00 & 0.00 & 0.00 & 0.00 & 0.00 \\
\hline $\mathrm{Ba}$ & 0.00 & 0.00 & 0.00 & 0.00 & 0.00 & 0.00 & 0.00 & 0.00 \\
\hline $\mathrm{Pb}$ & 0.00 & 0.00 & 0.00 & 0.00 & 0.00 & 0.00 & 0.00 & 0.00 \\
\hline $\mathrm{Fe}$ & 0.00 & 0.01 & 0.00 & 0.00 & 0.00 & 0.00 & 0.00 & 0.00 \\
\hline $\mathrm{Mn}$ & 0.00 & 0.00 & 0.00 & 0.00 & 0.00 & 0.00 & 0.00 & 0.00 \\
\hline Str & 0.82 & 0.61 & 0.64 & 0.57 & 0.57 & 0.56 & 0.56 & 0.67 \\
\hline $\mathrm{Ar}$ & 0.18 & 0.38 & 0.36 & 0.43 & 0.43 & 0.44 & 0.44 & 0.33 \\
\hline
\end{tabular}

Selected orthorhombic carbonate analyses from marble sample BA9/91 A. Str = strontianite, $\mathrm{Ar}=$ aragonite.

Table 4. Diffraction pattern for orthorhombic carbonate in calcite matrix.

\begin{tabular}{|c|c|c|c|c|}
\hline Sin theta & $\mathrm{d} \AA$ & $\mathrm{Cc}$ Int & Ar Int & hkl \\
\hline 0.3971 & 3.807 & 10 & & 012 \\
\hline 0.4337 & 3.480 & & 100 & 111 \\
\hline 0.4462 & 3.381 & & 60 & 021 \\
\hline 0.4955 & 3.037 & 100 & & 104 \\
\hline 0.5344 & 2.810 & 5 & & 006 \\
\hline 0.5934 & 2.523 & & 20 & 200 \\
\hline 0.5990 & 2.499 & 10 & & 110 \\
\hline 0.6115 & 2.446 & & 20 & 112 \\
\hline 0.6388 & 2.339 & 15 & & 113 \\
\hline 0.7431 & 1.9998 & 7 & & 202 \\
\hline 0.9063 & 1.6263 & 4 & & 211 \\
\hline
\end{tabular}

$\mathrm{Cc}$ Int $=$ intensity of calcite lines; Ar Int $=$ intensity of aragonite lines.

$60 \mathrm{~s}$, the LREE, Th, Y and $\mathrm{Zr}$ were all measured for $100 \mathrm{~s}$, and all other elements were measured for a count time of $30 \mathrm{~s}$. The carbonates and micas were analysed using a rastered beam $\left(3 \mu \mathrm{m}^{2}\right)$; all other minerals were analysed with a focused beam in spot mode.

Standards for $\mathrm{La}, \mathrm{Ce}, \mathrm{Pr}, \mathrm{Nd}, \mathrm{Sm}$ and Gd were pure synthetic phosphates of each element; the Th standard was natural thorite and the $\mathrm{Y}$ standard was a synthetic yttrium aluminium garnet (YAG: $\mathrm{Y}_{3} \mathrm{Al}_{5} \mathrm{O}_{12}$ ). The data for LREE were corrected for interelement interferences based on the methods and analyses presented in DeWolf (1993). Wavelength scans were used to detect the presence of minor elements. Relative errors are based on counting statistics for the standards and samples; for epidote and titanite these are c. $1 \%$ of amount present (OAP) for $\mathrm{Si}, \mathrm{Al}$ and $\mathrm{Ca}$, and c. $5-15 \%$ OAP for Sr, Th, Y and LREEs. In general the larger errors are associated with lower abundances of the minor elements and of LREEs.

Whole rock chemistry was analysed using a Philips PW 1400 XRF spectrometer equipped with a Sc-Mo tube at the University of Lausanne, Switzerland. For the $\mathrm{Sr}$ isotope analyses small fragments (1-5 mg) of carbonate were separated and dissolved in HCl. Subsequently $\mathrm{Sr}$ was separated with $2.5 \mathrm{~N} \mathrm{HCl}$ from the matrix columns filled with c. $15 \mathrm{~mL}$ DOWEX AG $50 \mathrm{Wx} 8^{\circledR}(200-400 \mathrm{mesh})$ resin. Strontium was measured on W-single central filaments in the dynamic mode. The sample was loaded with a slurry containing $\mathrm{TaF}_{5}$. Total procedural blank concentrations are negligible with less than $50 \mathrm{pg}$. Replicate measurements of NBS 987 standard yielded a mean ${ }^{87} \mathrm{Sr} /{ }^{86} \mathrm{Sr}$ value of $0.710265 \pm 20$. Fractionation was corrected by normalizing the $\mathrm{Sr}$-isotope ratios to ${ }^{86} \mathrm{Sr} /{ }^{88} \mathrm{Sr}=0.1194$. All isotope measurements were performed at the Zentrallaboratorium für Geochronologie at the Universität Münster.

\section{MINERAL COMPOSITIONS}

\section{Carbonates}

Calcite is the major phase in the marbles. Typically calcite within an individual sample is homogenous but there are slight compositional differences between samples (Table 2, Fig. 4a). Calcite contains 0.2-1.2 mole percentage $\mathrm{SrCO}_{3}$. Calcite from the Sjurdholmen samples is more enriched in $\mathrm{Fe}$ than those from Liavatn. Minor dolomite was found in only one marble sample (BA9/91 A). The dolomite occurs as $<1 \mu \mathrm{m}$ blebs in the calcite, and thus could not be analysed accurately with the electron microprobe. Carbonate inclusions in garnet from sample BA21/93 contain up to several weight percentage more $\mathrm{Mg}, \mathrm{Fe}$, and $\mathrm{Sr}$ than the adjacent calcite groundmass (Table 2).

One marble (BA9/91 A) contains minor amounts of a second $\mathrm{Ca}-\mathrm{Sr}$ rich carbonate that appears as small blebs throughout the groundmass and typically near internal fractures in the rock. The composition of this carbonate is intermediate between aragonite (Ar) and strontianite (Str), ranging from $\mathrm{Str}_{82}$ to $\mathrm{Str}_{56}$ (Table 3). Figure 5 is a ternary plot of these carbonates as well as other data from the literature. The compositions from this study confirm a greater extent of solid solution along the $\mathrm{CaCO}_{3}-\mathrm{SrCO}_{3}$ binary than was observed by Speer (1983), as was also concluded by Vorob'yev et al. (1989) and Pisarskii et al. (1998). The carbonate analyses from Vorob'yev et al. (1989) and Pisarskii et al. (1998) are suspect, however, because they appear to be finely intergrown with other carbonates, some showing complex exsolution textures. Additionally, they have not been adequately characterized structurally. 


\begin{tabular}{|c|c|c|c|c|c|c|c|c|}
\hline & $\begin{array}{l}\text { BA9/91 A' } \\
\mathrm{cpx} 1 / 4\end{array}$ & $\begin{array}{c}\text { Liavatn } \\
\mathrm{cpx} 1 / 1\end{array}$ & $\begin{array}{l}\text { BA21/93 } \\
\text { сpx } 5\end{array}$ & $\begin{array}{c}\text { BA21/93 } \\
\text { cpx } 9\end{array}$ & $\begin{array}{c}\text { BA25/92 A } \\
\text { cpx } 1 / 2\end{array}$ & $\begin{array}{c}\text { BA25/92B } \\
\mathrm{cp} 33 / 1\end{array}$ & $\begin{array}{c}\text { BA69/93 } \\
\text { сpx } 2 / 3\end{array}$ & $\begin{array}{c}\text { BA } 70 / 93 \\
\mathrm{cpx} 1 / 2\end{array}$ \\
\hline $\mathrm{SiO}_{2}$ & 53.21 & 53.08 & 53.67 & 52.79 & 52.32 & 52.74 & 54.28 & 52.61 \\
\hline $\mathrm{TiO}_{2}$ & 0.31 & 0.22 & 0.16 & 0.31 & 0.07 & 0.04 & 0.26 & 0.07 \\
\hline $\mathrm{Al}_{2} \mathrm{O}_{3}$ & 7.88 & 7.92 & 8.27 & 8.66 & 1.94 & 2.20 & 8.29 & 3.70 \\
\hline $\mathrm{Cr}_{2} \mathrm{O}_{3}$ & 0.05 & 0.01 & 0.00 & 0.02 & 0.04 & 0.04 & 0.00 & 0.00 \\
\hline $\mathrm{Fe}_{2} \mathrm{O}_{3}$ & 3.24 & 4.97 & 6.02 & 7.76 & 3.85 & 4.71 & 6.53 & 4.55 \\
\hline $\mathrm{FeO}$ & 6.88 & 5.18 & 4.61 & 3.72 & 9.70 & 8.18 & 5.97 & 8.61 \\
\hline $\mathrm{MnO}$ & 0.09 & 0.06 & 0.06 & 0.04 & 0.25 & 0.23 & 0.08 & 0.24 \\
\hline $\mathrm{MgO}$ & 7.53 & 7.69 & 8.13 & 7.78 & 9.15 & 9.07 & 5.66 & 8.55 \\
\hline $\mathrm{CaO}$ & 15.65 & 14.89 & 14.14 & 14.19 & 20.13 & 19.11 & 11.64 & 18.11 \\
\hline $\mathrm{Na}_{2} \mathrm{O}$ & 5.06 & 5.53 & 5.84 & 5.95 & 2.28 & 3.03 & 7.35 & 3.38 \\
\hline Total & 99.89 & 99.55 & 100.90 & 101.22 & 99.74 & 99.34 & 100.07 & 99.82 \\
\hline \multicolumn{9}{|c|}{ Calculated on the basis of 4 cations } \\
\hline $\mathrm{Si}$ & 1.96 & 1.95 & 1.94 & 1.91 & 1.98 & 1.99 & 1.99 & 1.98 \\
\hline $\mathrm{Al}^{\mathrm{iv}}$ & 0.04 & 0.05 & 0.06 & 0.09 & 0.02 & 0.01 & 0.01 & 0.02 \\
\hline $\mathrm{Al}^{\mathrm{vi}}$ & 0.30 & 0.29 & 0.29 & 0.28 & 0.07 & 0.09 & 0.34 & 0.14 \\
\hline $\mathrm{Fe}^{+3}$ & 0.09 & 0.14 & 0.17 & 0.21 & 0.11 & 0.13 & 0.18 & 0.13 \\
\hline $\mathrm{Ti}$ & 0.01 & 0.01 & 0.00 & 0.01 & 0.00 & 0.00 & 0.01 & 0.00 \\
\hline $\mathrm{Cr}$ & 0.00 & 0.00 & 0.00 & 0.00 & 0.00 & 0.00 & 0.00 & 0.00 \\
\hline $\mathrm{Mg}$ & 0.41 & 0.42 & 0.44 & 0.42 & 0.52 & 0.51 & 0.31 & 0.48 \\
\hline $\mathrm{Fe}^{+2}$ & 0.21 & 0.16 & 0.14 & 0.11 & 0.31 & 0.26 & 0.18 & 0.27 \\
\hline $\mathrm{Mn}$ & 0.00 & 0.00 & 0.00 & 0.00 & 0.01 & 0.01 & 0.00 & 0.01 \\
\hline $\mathrm{Ca}$ & 0.62 & 0.59 & 0.55 & 0.55 & 0.82 & 0.77 & 0.46 & 0.73 \\
\hline $\mathrm{Na}$ & 0.36 & 0.39 & 0.41 & 0.42 & 0.17 & 0.22 & 0.52 & 0.25 \\
\hline Aug & 0.63 & 0.60 & 0.57 & 0.57 & 0.83 & 0.77 & 0.47 & 0.75 \\
\hline Jd & 0.30 & 0.29 & 0.21 & 0.22 & 0.07 & 0.09 & 0.34 & 0.14 \\
\hline Aeg & 0.06 & 0.10 & 0.17 & 0.21 & 0.10 & 0.13 & 0.18 & 0.11 \\
\hline
\end{tabular}

Abrreviations: Aeg = aegirine: Aug = augite; c, core; cpx, clinopyroxene; $\mathrm{Jd}=$ jadeite; $r$, rim.

\begin{tabular}{|c|c|c|c|c|c|c|c|c|}
\hline & $\begin{array}{c}\mathrm{BA} 21 / 93 \\
\text { garl/3c }\end{array}$ & $\begin{array}{l}\text { BA21/93 } \\
\text { gar1/11r }\end{array}$ & $\begin{array}{c}\mathrm{BA} 21 / 93 \\
\operatorname{gar} 2 / 3 \mathrm{r}\end{array}$ & $\begin{array}{c}\mathrm{BA} 21 / 93 \\
\operatorname{gar} 2 / 5 \mathrm{c}\end{array}$ & $\begin{array}{l}\text { Liavatn } \\
\text { garl } 1 / 1 \mathrm{c}\end{array}$ & $\begin{array}{l}\text { Liavatn } \\
\text { garl/3r }\end{array}$ & $\begin{array}{c}\text { BA } 69 / 93 \\
\text { gar } 1 / 1 \mathrm{c}\end{array}$ & $\begin{array}{c}\text { BA } 69 / 93 \\
\operatorname{gar} 1 / 2 \mathrm{r}\end{array}$ \\
\hline $\mathrm{SiO}_{2}$ & 37.91 & 37.74 & 38.23 & 38.05 & 37.92 & 38.33 & 37.55 & 37.63 \\
\hline $\mathrm{TiO}_{2}$ & 0.38 & 0.08 & 0.10 & 0.46 & 0.14 & 0.07 & 0.05 & 0.05 \\
\hline $\mathrm{Al}_{2} \mathrm{O}_{3}$ & 20.65 & 20.58 & 21.25 & 20.84 & 20.46 & 21.11 & 20.42 & 20.60 \\
\hline $\mathrm{Cr}_{2} \mathrm{O}_{3}$ & 0.01 & 0.02 & 0.02 & 0.03 & 0.00 & 0.00 & 0.00 & 0.00 \\
\hline $\mathrm{FeO}$ & 24.64 & 26.08 & 25.43 & 24.31 & 26.30 & 24.99 & 27.99 & 26.36 \\
\hline $\mathrm{MnO}$ & 1.17 & 1.04 & 0.87 & 1.04 & 0.98 & 0.82 & 2.86 & 2.79 \\
\hline $\mathrm{MgO}$ & 2.94 & 2.99 & 4.17 & 3.79 & 3.05 & 3.55 & 2.09 & 2.14 \\
\hline $\mathrm{CaO}$ & 11.66 & 10.76 & 10.07 & 11.38 & 11.24 & 11.18 & 9.57 & 9.87 \\
\hline Total & 99.38 & 99.29 & 100.13 & 99.90 & 100.08 & 100.04 & 100.52 & 99.45 \\
\hline \multicolumn{9}{|c|}{ Calculated on the basis of 12 oxygen } \\
\hline $\mathrm{Si}$ & 3.01 & 3.01 & 2.99 & 2.98 & 3.00 & 3.01 & 2.99 & 3.01 \\
\hline $\mathrm{Al}$ & 1.93 & 1.93 & 1.96 & 1.93 & 1.91 & 1.95 & 1.92 & 1.94 \\
\hline $\mathrm{Ti}$ & 0.02 & 0.00 & 0.01 & 0.03 & 0.01 & 0.00 & 0.00 & 0.00 \\
\hline $\mathrm{Cr}$ & 0.00 & 0.00 & 0.00 & 0.00 & 0.00 & 0.00 & 0.00 & 0.00 \\
\hline $\mathrm{Mg}$ & 0.35 & 0.35 & 0.49 & 0.44 & 0.36 & 0.42 & 0.25 & 0.26 \\
\hline $\mathrm{Fe}^{+2}$ & 1.63 & 1.74 & 1.66 & 1.59 & 1.74 & 1.64 & 1.87 & 1.76 \\
\hline $\mathrm{Mn}$ & 0.08 & 0.07 & 0.06 & 0.07 & 0.07 & 0.05 & 0.19 & 0.19 \\
\hline $\mathrm{Ca}$ & 0.99 & 0.92 & 0.84 & 0.96 & 0.95 & 0.94 & 0.82 & 0.85 \\
\hline $\mathrm{O}$ & 12.00 & 12.00 & 12.00 & 12.00 & 12.00 & 12.00 & 12.00 & 12.00 \\
\hline Alm & 53.55 & 56.40 & 53.69 & 51.23 & 55.80 & 53.76 & 59.71 & 57.74 \\
\hline Grs & 32.43 & 29.76 & 28.16 & 31.76 & 30.54 & 30.83 & 26.16 & 27.70 \\
\hline Pyr & 11.39 & 11.51 & 16.24 & 14.70 & 11.55 & 13.63 & 7.94 & 8.36 \\
\hline Sps & 2.58 & 2.28 & 1.91 & 2.30 & 2.11 & 1.78 & 6.19 & 6.19 \\
\hline Uv & 0.04 & 0.06 & 0.03 & 0.07 & 0.00 & 0.00 & 0.00 & 0.00 \\
\hline $\mathrm{Mg} / \mathrm{Mg}+\mathrm{Fe}$ & 0.18 & 0.17 & 0.23 & 0.22 & 0.17 & 0.20 & 0.12 & 0.13 \\
\hline
\end{tabular}

Abbreviations: Alm, almandine; c, core; Grs, grossular; Pyr, pryrope; r, rim; Sps, spessartine; Uv, uvarovite.

Single crystal XRD analysis was performed on a grain of the intermediate carbonate from sample BA9/91 A using a Gandolfi camera to determine cell parameters. Table 4 shows the diffraction pattern for a grain of intermediate composition $\operatorname{Str}_{60}$, as well as calcite peaks, which appear in the pattern because the orthorhombic carbonate could not be completely removed from the matrix. The $d$-values for the two strongest lines of the intermediate carbonate $(111$ \&
Table 6. Garnet analyses.

Table 5. Clinopyroxene analyses.
021) lie between the $d$-values for aragonite and strontianite (Table 4), 60\% toward strontianite. The experiments of Carlson (1980) suggest that $\operatorname{Str}_{56}-\operatorname{Str}_{67}$ is not stable with calcite at the peak conditions experienced by these marbles. Dasgupta et al. (1990) also noted the occurrence of calcian strontianite with compositions of $\operatorname{Str}_{71}-\operatorname{Str}_{61}$ in equilibrium with calcite and barytocalcite in metamorphic rocks (Fig. 5) and concluded that they do not fit with experimentally 
Table 7. Epidote and allanite analyses.

\begin{tabular}{|c|c|c|c|c|c|c|c|c|c|c|c|}
\hline & $\begin{array}{c}\mathrm{HA} 4 / 90 \\
2 / 1\end{array}$ & $\begin{array}{c}\mathrm{HA} 5 / 90 \\
3 / 1\end{array}$ & $\begin{array}{c}\text { BA } 9 / 91 \mathrm{~A} \\
3 / 1\end{array}$ & $\begin{array}{c}\mathrm{BA} 9 / 91 \mathrm{~A}^{\prime} \\
1 / 1 \mathrm{c}\end{array}$ & $\begin{array}{c}\text { BA } 9 / 92 \mathrm{~A} \\
1 / 2\end{array}$ & $\begin{array}{c}\text { BA9/92B } \\
1 / 1\end{array}$ & $\begin{array}{c}\text { BA21/93 } \\
1 / 1\end{array}$ & $\begin{array}{c}\mathrm{BA} 21 / 93 \\
1 / 8\end{array}$ & $\begin{array}{c}\mathrm{BA} 25 / 92 \mathrm{~A} \\
1 / 1\end{array}$ & $\begin{array}{c}\mathrm{BA} 25 / 92 \mathrm{~B} \\
1 / 14 \mathrm{r}\end{array}$ & $\begin{array}{c}\text { BA } 69 / 93 \\
2 / 1\end{array}$ \\
\hline $\mathrm{SiO}_{2}$ & 36.34 & 36.35 & 37.84 & 37.47 & 38.20 & 38.02 & 34.57 & 36.92 & 32.87 & 34.02 & 35.03 \\
\hline $\mathrm{TiO}_{2}$ & 0.10 & 0.17 & 0.36 & 0.33 & 0.11 & 0.24 & 0.28 & 0.34 & 0.23 & 0.28 & 0.25 \\
\hline $\mathrm{Al}_{2} \mathrm{O}_{3}$ & 25.60 & 24.56 & 26.53 & 25.70 & 27.43 & 26.63 & 23.38 & 26.59 & 19.42 & 20.14 & 22.05 \\
\hline $\mathrm{Fe}_{2} \mathrm{O}_{3}$ & 7.49 & 3.43 & 0.28 & 0.24 & -0.03 & 0.14 & 9.57 & 8.17 & 6.67 & 4.91 & 4.48 \\
\hline $\mathrm{FeO}$ & 0.82 & 5.15 & 7.46 & 7.91 & 6.64 & 6.21 & 0.00 & 0.00 & 6.34 & 7.52 & 6.60 \\
\hline $\mathrm{MnO}$ & 0.08 & 0.07 & 0.07 & 0.09 & 0.00 & 0.05 & 0.06 & 0.09 & 0.07 & 0.15 & 0.16 \\
\hline $\mathrm{MgO}$ & 0.23 & 0.36 & 0.20 & 0.21 & 0.09 & 0.20 & 0.49 & 0.18 & 0.53 & 0.43 & 0.49 \\
\hline $\mathrm{CaO}$ & 19.97 & 18.89 & 21.86 & 21.75 & 22.98 & 21.70 & 18.01 & 21.49 & 15.55 & 16.71 & 17.40 \\
\hline $\mathrm{SrO}$ & 2.56 & 1.98 & 1.61 & 1.53 & 2.03 & 1.58 & 2.00 & 2.15 & 0.88 & 0.72 & 1.05 \\
\hline $\mathrm{Y}_{2} \mathrm{O}_{3}$ & 0.17 & 0.10 & 0.00 & 0.00 & 0.06 & 0.00 & 0.00 & 0.00 & 0.00 & 0.02 & 0.10 \\
\hline $\mathrm{La}_{2} \mathrm{O}_{3}$ & 1.34 & 2.33 & 0.15 & 0.33 & 0.07 & 0.30 & 2.53 & 0.12 & 3.65 & 2.71 & 2.30 \\
\hline $\mathrm{Ce}_{2} \mathrm{O}_{3}$ & 2.18 & 2.99 & 0.52 & 0.67 & 0.00 & 0.50 & 3.62 & 0.29 & 6.71 & 4.89 & 4.22 \\
\hline $\mathrm{Pr}_{2} \mathrm{O}_{3}$ & 0.08 & 0.48 & 0.07 & 0.00 & 0.04 & 0.00 & 0.98 & 0.08 & 0.72 & 0.44 & 0.43 \\
\hline $\mathrm{Nd}_{2} \mathrm{O}_{3}$ & 0.65 & 0.90 & 0.39 & 0.29 & 0.05 & 0.07 & 1.19 & 0.21 & 2.12 & 1.78 & 2.24 \\
\hline $\mathrm{Sm}_{2} \mathrm{O}_{3}$ & 0.00 & 0.10 & 0.07 & 0.01 & 0.00 & 0.00 & 0.56 & 0.04 & 0.36 & 0.60 & 0.33 \\
\hline $\mathrm{Gd}_{2} \mathrm{O}_{3}$ & 0.16 & 0.41 & 0.23 & 0.02 & 0.00 & 0.00 & 0.23 & 0.18 & 0.00 & 0.00 & 0.03 \\
\hline $\mathrm{ThO}_{2}$ & 0.12 & 0.00 & 0.06 & 0.01 & 0.12 & 0.00 & 0.00 & 0.00 & 0.05 & 0.09 & 0.30 \\
\hline $\mathrm{ZrO}_{2}$ & 0.00 & 0.04 & 0.05 & 0.00 & 0.00 & 0.02 & 0.00 & 0.00 & 0.00 & 0.01 & 0.00 \\
\hline $\mathrm{F}$ & 0.00 & 0.05 & 0.04 & 0.00 & 0.00 & 0.02 & 0.00 & 0.00 & 0.10 & 0.01 & 0.03 \\
\hline Total & 97.91 & 98.35 & 97.78 & 96.55 & 97.80 & 95.70 & 97.45 & 96.84 & 96.27 & 95.46 & 97.50 \\
\hline \multicolumn{12}{|c|}{ Calc. on basis of 8 cations } \\
\hline $\mathrm{Si}$ & 2.97 & 2.99 & 2.99 & 3.00 & 2.99 & 3.05 & 3.00 & 3.00 & 2.93 & 2.99 & 2.98 \\
\hline $\mathrm{Ti}$ & 0.01 & 0.01 & 0.02 & 0.02 & 0.01 & 0.01 & 0.02 & 0.02 & 0.02 & 0.02 & 0.02 \\
\hline $\mathrm{Al}$ & 2.46 & 2.38 & 2.47 & 2.43 & 2.53 & 2.52 & 2.39 & 2.55 & 2.04 & 2.09 & 2.21 \\
\hline $\mathrm{Fe}^{+3}$ & 0.41 & 0.41 & 0.48 & 0.49 & 0.43 & 0.35 & 0.62 & 0.50 & 0.66 & 0.55 & 0.49 \\
\hline $\mathrm{Fe}^{+2}$ & 0.12 & 0.15 & 0.03 & 0.05 & 0.00 & 0.08 & 0.00 & 0.00 & 0.25 & 0.32 & 0.26 \\
\hline $\mathrm{Mg}$ & 0.01 & 0.00 & 0.00 & 0.01 & 0.00 & 0.00 & 0.06 & 0.02 & 0.01 & 0.01 & 0.01 \\
\hline $\mathrm{Mn}$ & 0.03 & 0.04 & 0.02 & 0.02 & 0.01 & 0.02 & 0.00 & 0.01 & 0.07 & 0.06 & 0.06 \\
\hline $\mathrm{Ca}$ & 1.75 & 1.67 & 1.85 & 1.87 & 1.93 & 1.86 & 1.67 & 1.87 & 1.49 & 1.57 & 1.59 \\
\hline $\mathrm{Sr}$ & 0.12 & 0.09 & 0.07 & 0.07 & 0.09 & 0.07 & 0.10 & 0.10 & 0.05 & 0.04 & 0.05 \\
\hline $\mathrm{Y}$ & 0.01 & 0.00 & 0.00 & 0.00 & 0.00 & 0.00 & 0.00 & 0.00 & 0.00 & 0.00 & 0.00 \\
\hline REE & 0.14 & 0.23 & 0.04 & 0.04 & 0.00 & 0.03 & 0.29 & 0.03 & 0.46 & 0.35 & 0.31 \\
\hline $\mathrm{Th}$ & 0.00 & 0.00 & 0.00 & 0.00 & 0.00 & 0.00 & 0.00 & 0.00 & 0.00 & 0.00 & 0.01 \\
\hline $\mathrm{Zr}$ & 0.00 & 0.00 & 0.00 & 0.00 & 0.00 & 0.00 & 0.00 & 0.00 & 0.00 & 0.00 & 0.00 \\
\hline $\mathrm{F}$ & 0.00 & 0.01 & 0.01 & 0.00 & 0.00 & 0.01 & 0.00 & 0.00 & 0.03 & 0.00 & 0.01 \\
\hline sum oct ${ }^{1}$ & 2.99 & 2.96 & 3.01 & 3.00 & 2.97 & 2.96 & 3.10 & 3.09 & 2.97 & 2.98 & 2.99 \\
\hline $\operatorname{sum} \mathrm{A}^{2}$ & 2.05 & 2.04 & 1.99 & 2.00 & 2.04 & 1.99 & 2.07 & 2.00 & 2.07 & 2.02 & 2.02 \\
\hline
\end{tabular}

${ }^{1}$ sum oct $=\mathrm{Al}+\mathrm{Fe}^{3+}+\mathrm{Fe}^{2+}+\mathrm{Mg}+\mathrm{Ti}^{2}{ }^{2}$ sum $\mathrm{A}=\mathrm{Ca}+\mathrm{Mn}+\mathrm{Ba}+\mathrm{Sr}+\mathrm{Y}+\mathrm{REE}+\mathrm{Th}+\mathrm{Zr}$.

determined stability fields. Extrapolation of Carlson's isopleths suggests that strontianite compositions $\left(\mathrm{Str}_{56}\right.$ $\operatorname{Str}_{67}$ ) may become stable with calcite at much lower temperatures and pressures. For example, strontianite with composition $\operatorname{Str}_{60}$ would be in equilibrium with calcite at approximately $300{ }^{\circ} \mathrm{C}$ and $2 \mathrm{kbar}$. These data imply that carbonate compositions may record late recrystallization during exhumation of the marbles. However, Casey et al. (1996) predicted from dropsolution enthalpies that intermediate compositions are thermodynamically unstable and should unmix to form calcic strontianite and strontian aragonite at low temperatures. The miscibility gap should widen if calcite is formed instead of aragonite. Using a $W=$ $13.5 \mathrm{~kJ} \mathrm{~mol}^{-1}$, the crest of a symmetrical miscibility gap $\left(\mathrm{Str}_{50} \mathrm{Ar}_{50}\right)$ is predicted to be at about $540{ }^{\circ} \mathrm{C}$. This crest lies very close to our $\mathrm{Str}_{56}$ composition, and the predicted temperature agrees with our thermometric estimates of peak metamorphic conditions. More experiments are needed in the system $\mathrm{CaCO}_{3}-\mathrm{SrCO}_{3}$ to confirm the presence of the solvus and to enable extrapolation of compositions of coexisting calcite and calcian strontianite over a wider range of $P-T$.

\section{Clinopyroxene}

Clinopyroxene in the marbles has similar compositions to those in adjacent eclogite samples. Liavatn marbles contain clinopyroxene with compositions of $\mathrm{Jd}_{29-31}$, whereas an adjacent sample from an eclogite layer has a composition of $\mathrm{Jd}_{29-32}$ (Table 5, Fig. 6). Garnet from a Liavatn calc-silicate contains inclusions of omphacitic clinopyroxene $\left(\mathrm{Jd}_{22}\right)$. Marbles from the Sjurdholmen locality have clinopyroxene that is more diopsidic with only a few mole percent jadeite $\left(\mathrm{Jd}_{6-14}\right)$. Clinopyroxene from an adjacent eclogite sample has similar low jadeite contents $\left(\mathrm{Jd}_{14-17}\right)$, whereas another has a higher jadeite content $\left(\mathrm{Jd}_{33-34}\right)$ (Table 5). No $\mathrm{K}_{2} \mathrm{O}$ was detected in any of the clinopyroxene. Eclogites from the major eclogite facies shear zones have clinopyroxene compositions of $\mathrm{Jd}_{39-49}$ and the protoliths are gabbroic anorthosites to gabbros (Austrheim \& Griffin, 1985; Boundy et al., 1992). The difference in jadeite content may well be a function of bulk composition rather than $P-T$ conditions. 
656 T. M. BOUNDY ET AL.

Table 8. Titanite analyses.

\begin{tabular}{|c|c|c|c|c|c|c|c|c|c|c|}
\hline $\mathrm{Wt} \%$ Oxide & $\begin{array}{c}\mathrm{HA} 5 / 90 \\
1 / 1 \mathrm{c}\end{array}$ & $\begin{array}{c}\mathrm{HA} 5 / 90 \\
2 / 1\end{array}$ & $\begin{array}{c}\text { BA } 9 / 91 \mathrm{~A}^{\prime} \\
2 / 1\end{array}$ & $\begin{array}{c}\mathrm{BA} 9 / 91 \mathrm{~A}^{\prime} \\
2 / 2 \mathrm{r}\end{array}$ & $\begin{array}{c}\mathrm{BA} 25 / 92 \mathrm{~A}^{\prime} \\
1 / 1 \mathrm{c}\end{array}$ & $\begin{array}{c}\mathrm{BA} 25 / 92 \mathrm{~A}^{\prime} \\
1 / 2 \mathrm{r}\end{array}$ & $\begin{array}{c}\mathrm{BA} 25 / 92 \mathrm{~B} \\
1 / 1 \mathrm{c}\end{array}$ & $\begin{array}{c}\mathrm{BA} 25 / 92 \mathrm{~B} \\
1 / 2 \mathrm{r}\end{array}$ & $\begin{array}{c}\text { Liavatn } \\
1 / 1\end{array}$ & $\begin{array}{c}\text { Liavatn } \\
2 / 1\end{array}$ \\
\hline $\mathrm{SiO}_{2}$ & 30.61 & 30.41 & 30.26 & 29.62 & 30.10 & 29.96 & 30.53 & 30.09 & 30.51 & 30.67 \\
\hline $\mathrm{TiO}_{2}$ & 34.65 & 34.15 & 35.02 & 35.06 & 35.18 & 35.28 & 35.88 & 35.81 & 35.78 & 36.24 \\
\hline $\mathrm{Al}_{2} \mathrm{O}_{3}$ & 2.52 & 2.70 & 2.47 & 2.29 & 0.94 & 0.76 & 1.43 & 1.38 & 2.32 & 1.67 \\
\hline $\mathrm{Fe}_{2} \mathrm{O}_{3}$ & 0.70 & 0.81 & 0.60 & 0.65 & 2.09 & 1.82 & 1.06 & 0.84 & 0.49 & 0.49 \\
\hline $\mathrm{MnO}$ & 0.05 & 0.03 & 0.05 & 0.05 & 0.06 & 0.04 & 0.01 & 0.03 & 0.02 & 0.04 \\
\hline $\mathrm{MgO}$ & 0.00 & 0.00 & 0.01 & 0.00 & 0.01 & 0.01 & 0.02 & 0.02 & 0.00 & 0.00 \\
\hline $\mathrm{CaO}$ & 28.43 & 28.23 & 28.78 & 28.83 & 27.93 & 28.13 & 27.78 & 28.09 & 28.11 & 28.47 \\
\hline $\mathrm{BaO}$ & 0.00 & 0.00 & 0.00 & 0.00 & 0.00 & 0.00 & 0.00 & 0.00 & 0.00 & 0.00 \\
\hline $\mathrm{SrO}$ & 0.30 & 0.82 & 0.05 & 0.13 & 0.33 & 0.22 & 0.00 & 0.11 & 0.24 & 0.21 \\
\hline $\mathrm{Y}_{2} \mathrm{O}_{3}$ & 0.48 & 0.00 & 0.03 & 0.04 & 0.05 & 0.06 & 0.29 & 0.18 & 0.00 & 0.00 \\
\hline $\mathrm{La}_{2} \mathrm{O}_{3}$ & 0.02 & 0.02 & 0.00 & 0.00 & 0.05 & 0.00 & 0.00 & 0.01 & 0.03 & 0.00 \\
\hline $\mathrm{Ce}_{2} \mathrm{O}_{3}$ & 0.00 & 0.00 & 0.11 & 0.09 & 0.22 & 0.31 & 0.16 & 0.08 & 0.12 & 0.21 \\
\hline $\mathrm{Pr}_{2} \mathrm{O}_{3}$ & 0.11 & 0.06 & 0.00 & 0.00 & 0.00 & 0.15 & 0.00 & 0.00 & 0.14 & 0.11 \\
\hline $\mathrm{Nd}_{2} \mathrm{O}_{3}$ & 0.06 & 0.15 & 0.00 & 0.00 & 0.21 & 0.18 & 0.15 & 0.29 & 0.17 & 0.19 \\
\hline $\mathrm{Sm}_{2} \mathrm{O}_{3}$ & 0.06 & 0.03 & 0.00 & 0.00 & 0.04 & 0.05 & 0.18 & 0.12 & 0.00 & 0.00 \\
\hline $\mathrm{Gd}_{2} \mathrm{O}_{3}$ & 0.00 & 0.00 & 0.27 & 0.03 & 0.08 & 0.13 & 0.12 & 0.00 & 0.05 & 0.18 \\
\hline $\mathrm{ThO}_{2}$ & 0.00 & 0.01 & 0.10 & 0.07 & 0.00 & 0.00 & 0.08 & 0.06 & 0.00 & 0.00 \\
\hline $\mathrm{ZrO}_{2}$ & 0.02 & 0.00 & 0.00 & 0.02 & 0.10 & 0.00 & 0.04 & 0.11 & 0.03 & 0.06 \\
\hline $\mathrm{F}$ & 0.34 & 0.33 & 0.38 & 0.40 & 0.40 & 0.22 & 0.27 & 0.29 & 0.17 & 0.19 \\
\hline Total & 98.36 & 97.74 & 98.14 & 97.29 & 97.79 & 97.33 & 98.01 & 97.52 & 98.17 & 98.73 \\
\hline \multicolumn{11}{|c|}{ Calc. on basis of 3 cations } \\
\hline $\mathrm{Si}$ & 1.00 & 0.99 & 0.98 & 0.97 & 0.99 & 1.00 & 1.01 & 0.99 & 1.00 & 1.00 \\
\hline $\mathrm{Ti}$ & 0.85 & 0.84 & 0.86 & 0.86 & 0.87 & 0.88 & 0.89 & 0.89 & 0.88 & 0.89 \\
\hline $\mathrm{Al}$ & 0.10 & 0.10 & 0.09 & 0.09 & 0.04 & 0.03 & 0.06 & 0.05 & 0.09 & 0.06 \\
\hline $\mathrm{Fe}^{+3}$ & 0.02 & 0.02 & 0.01 & 0.02 & 0.05 & 0.05 & 0.03 & 0.02 & 0.01 & 0.01 \\
\hline $\mathrm{Mg}$ & 0.00 & 0.00 & 0.00 & 0.00 & 0.00 & 0.00 & 0.00 & 0.00 & 0.00 & 0.00 \\
\hline $\mathrm{Mn}$ & 0.00 & 0.00 & 0.00 & 0.00 & 0.00 & 0.00 & 0.00 & 0.00 & 0.00 & 0.00 \\
\hline $\mathrm{Ca}$ & 0.99 & 0.99 & 1.00 & 1.01 & 0.99 & 1.00 & 0.98 & 0.99 & 0.99 & 1.00 \\
\hline $\mathrm{Ba}$ & 0.00 & 0.00 & 0.00 & 0.00 & 0.00 & 0.00 & 0.00 & 0.00 & 0.00 & 0.00 \\
\hline $\mathrm{Sr}$ & 0.01 & 0.02 & 0.00 & 0.00 & 0.01 & 0.00 & 0.00 & 0.00 & 0.00 & 0.00 \\
\hline $\mathrm{Y}$ & 0.01 & 0.00 & 0.00 & 0.00 & 0.00 & 0.00 & 0.01 & 0.00 & 0.00 & 0.00 \\
\hline REE & 0.00 & 0.00 & 0.00 & 0.00 & 0.01 & 0.01 & 0.01 & 0.01 & 0.01 & 0.01 \\
\hline $\mathrm{Th}$ & 0.00 & 0.00 & 0.00 & 0.00 & 0.00 & 0.00 & 0.00 & 0.00 & 0.00 & 0.00 \\
\hline $\mathrm{Zr}$ & 0.00 & 0.00 & 0.00 & 0.00 & 0.00 & 0.00 & 0.00 & 0.00 & 0.00 & 0.00 \\
\hline $\mathrm{F}$ & 0.03 & 0.03 & 0.04 & 0.04 & 0.04 & 0.02 & 0.03 & 0.03 & 0.02 & 0.02 \\
\hline $\operatorname{sum} A$ & 1.01 & 1.01 & 1.01 & 1.02 & 1.00 & 1.02 & 1.00 & 1.01 & 1.00 & 1.01 \\
\hline sum oct & 0.96 & 0.96 & 0.97 & 0.97 & 0.96 & 0.96 & 0.97 & 0.97 & 0.98 & 0.97 \\
\hline
\end{tabular}

Table 9. Celestine and barite analyses.

\begin{tabular}{|c|c|c|c|c|c|c|c|c|c|c|}
\hline & $\begin{array}{l}\text { HA4/90 } \\
\text { cel } 1 / 1\end{array}$ & $\begin{array}{c}\text { BA9/91 A } \\
\text { cel } 1 / 6\end{array}$ & $\begin{array}{c}\text { BA9/91 A } \\
\text { cel } 2 / 3\end{array}$ & $\begin{array}{c}\text { BA9/91 A } \\
\text { int } 3 / 7\end{array}$ & $\begin{array}{c}\mathrm{BA} 9 / 91 \mathrm{~A}^{\prime} \\
\text { cel } 2 / 2\end{array}$ & $\begin{array}{c}\text { BA9 } 9 / 91 \mathrm{~A}^{\prime} \\
\text { bar } 2 / 2\end{array}$ & $\begin{array}{c}\mathrm{BA} 25 / 92 \mathrm{~A} \\
\text { cel } 1 / 1 \mathrm{a}\end{array}$ & $\begin{array}{c}\text { BA25/92 A } \\
\text { bar } 4 / 1\end{array}$ & $\begin{array}{c}\mathrm{BA} 25 / 92 \mathrm{~B} \\
\text { cel } 5 / 2\end{array}$ & $\begin{array}{c}\text { BA25/92B } \\
\text { bar } 3 / 1\end{array}$ \\
\hline $\mathrm{SO}_{3}$ & 43.78 & 40.76 & 43.74 & 40.36 & 44.53 & 33.72 & 41.15 & 33.43 & 40.24 & 33.04 \\
\hline $\mathrm{TiO}_{2}$ & 0.00 & 0.00 & 0.00 & 0.00 & 0.06 & 0.01 & 0.00 & 0.00 & 0.00 & 0.00 \\
\hline $\mathrm{SrO}$ & 48.55 & 57.16 & 51.59 & 23.65 & 55.59 & 2.98 & 35.94 & 4.82 & 35.49 & 2.21 \\
\hline $\mathrm{PbO}$ & 0.00 & 0.00 & 0.00 & 0.00 & 0.00 & 0.00 & 0.00 & 0.00 & 0.00 & 0.00 \\
\hline $\mathrm{CaO}$ & 3.19 & 0.46 & 0.09 & 0.32 & 0.22 & 0.10 & 3.25 & 0.17 & 2.20 & 0.92 \\
\hline $\mathrm{BaO}$ & 5.30 & 0.35 & 3.03 & 34.36 & 1.52 & 62.01 & 18.55 & 59.09 & 21.54 & 64.38 \\
\hline $\mathrm{FeO}$ & 0.04 & 0.06 & 0.04 & 0.01 & 0.03 & 0.39 & 0.14 & 0.00 & 0.07 & 0.01 \\
\hline $\mathrm{MnO}$ & 0.03 & 0.00 & 0.00 & 0.00 & 0.00 & 0.00 & 0.00 & 0.00 & 0.00 & 0.00 \\
\hline Total & 100.88 & 98.80 & 98.49 & 98.70 & 101.95 & 99.21 & 99.03 & 97.52 & 99.55 & 100.56 \\
\hline \multicolumn{11}{|c|}{ Calculated on the basis of 2 cations } \\
\hline S & 0.99 & 0.98 & 1.01 & 1.02 & 1.00 & 0.98 & 0.99 & 0.98 & 0.98 & 0.95 \\
\hline $\mathrm{Ti}$ & 0.00 & 0.00 & 0.00 & 0.00 & 0.00 & 0.00 & 0.00 & 0.00 & 0.00 & 0.00 \\
\hline $\mathrm{Sr}$ & 0.85 & 1.06 & 0.92 & 0.46 & 0.97 & 0.07 & 0.67 & 0.11 & 0.67 & 0.05 \\
\hline $\mathrm{Pb}$ & 0.00 & 0.00 & 0.00 & 0.00 & 0.00 & 0.00 & 0.00 & 0.00 & 0.00 & 0.00 \\
\hline $\mathrm{Ca}$ & 0.10 & 0.03 & 0.00 & 0.01 & 0.01 & 0.00 & 0.11 & 0.01 & 0.08 & 0.04 \\
\hline $\mathrm{Ba}$ & 0.06 & 0.01 & 0.04 & 0.45 & 0.02 & 0.94 & 0.23 & 0.90 & 0.27 & 0.96 \\
\hline $\mathrm{Fe}$ & 0.00 & 0.00 & 0.00 & 0.00 & 0.00 & 0.01 & 0.00 & 0.00 & 0.00 & 0.00 \\
\hline $\mathrm{Mn}$ & 0.00 & 0.00 & 0.00 & 0.00 & 0.00 & 0.00 & 0.00 & 0.00 & 0.00 & 0.00 \\
\hline $\mathrm{O}$ & 3.97 & 4.02 & 3.95 & 3.91 & 4.01 & 3.96 & 3.97 & 3.96 & 3.96 & 3.90 \\
\hline Celestine & 83.7 & 96.4 & 95.8 & 50.0 & 97.5 & 6.6 & 66.0 & 10.7 & 65.6 & 4.7 \\
\hline Barite & 6.2 & 0.9 & 4.2 & 48.9 & 1.8 & 93.0 & 23.0 & 88.6 & 26.9 & 91.7 \\
\hline Anhydrite & 10.2 & 2.7 & 0.0 & 1.0 & 0.7 & 0.4 & 11.0 & 0.7 & 7.5 & 3.6 \\
\hline
\end{tabular}

cel $=$ celestine, bar $=$ barite, int $=$ intermediate. 
Table 10. Biotite analysis.

\begin{tabular}{lrr}
\hline $\mathrm{Wt} \%$ Oxide & BA 25/92a.1 & BA $25 / 92 \mathrm{a} .2$ \\
\hline $\mathrm{Al}_{2} \mathrm{O}_{3}$ & 13.12 & 13.27 \\
$\mathrm{FeO}$ & 23.47 & 23.21 \\
$\mathrm{MnO}$ & 0.14 & 0.21 \\
$\mathrm{MgO}$ & 9.95 & 10.22 \\
$\mathrm{CaO}$ & 0.26 & 0.31 \\
$\mathrm{BaO}$ & 0.38 & 0.39 \\
$\mathrm{Na} 2 \mathrm{O}$ & 0.14 & 0.09 \\
$\mathrm{~K}_{2} \mathrm{O}$ & 8.82 & 8.31 \\
$\mathrm{~F}$ & 0.01 & 0.01 \\
$\mathrm{Cl}$ & 0.07 & 0.07 \\
$\mathrm{Total}$ & 94.70 & 94.83 \\
$\mathrm{Calc}$. on basis of 24 oxygen & & \\
$\mathrm{Si}$ & & 6.26 \\
$\mathrm{Ti}$ & 6.25 & 0.27 \\
$\mathrm{Al}$ & 0.26 & 2.67 \\
$\mathrm{Fe}$ & 3.31 \\
$\mathrm{Mn}$ & 2.66 & 0.03 \\
$\mathrm{Mg}$ & 3.37 & 2.60 \\
$\mathrm{Ca}$ & 0.02 & 0.06 \\
$\mathrm{Ba}$ & 2.55 & 0.03 \\
$\mathrm{Na}$ & 0.05 & 0.03 \\
$\mathrm{~K}$ & 0.03 & 1.81 \\
$\mathrm{~F}$ & 0.05 & 0.01 \\
$\mathrm{Cl}$ & 1.93 & 0.02 \\
$\mathrm{X}_{\mathrm{Phl}}$ & 0.01 & 0.44 \\
$\mathrm{X}_{\mathrm{Ann}}$ & 0.02 & 0.56 \\
\hline
\end{tabular}

\section{Garnet}

Garnet was found in only one of the carbonate samples, Liavatn sample BA21/93. The compositions vary from $\mathrm{Alm}_{52-56} \mathrm{Grs}_{28-33} \mathrm{Pyp}_{11-16} \mathrm{Sps}_{2}$ (Table 6) and show weak prograde zonation. Garnet from one of the adjacent eclogite layers displays similar compositions of $\mathrm{Alm}_{51-56} \mathrm{Grs}_{31-35} \mathrm{Pyp}_{10-14} \mathrm{Sps}_{2}$ excluding the core of one large garnet analysed, which may be relict from earlier granulite facies metamorphism (sample Liavatn; Table 6). The eclogite sample from Sjurdholmen (BA69/93) has similar garnet compositions of $\mathrm{Alm}_{58-60} \mathrm{Grs}_{26-28} \mathrm{Pyp}_{8} \mathrm{Sps}_{6}$ (Fig. 7). In contrast, eclogite facies garnet from the major shear zones is typically less almandine rich and much more pyropic $\left(\mathrm{Alm}_{40} \mathrm{Grs}_{21} \mathrm{Pyp}_{38} \mathrm{Sps}_{1}\right)$ for the anorthosite protolith and $\mathrm{Alm}_{47} \mathrm{Grs}_{26} \mathrm{Pyp}_{26} \mathrm{Sps}_{1}$ for the gabbro protolith; Boundy et al., 1992).

\section{Epidote/Allanite}

The Liavatn marbles contain REE-bearing epidote, whereas in the Sjurdholmen marbles it is more properly termed allanite (Table 7). In the investigated marbles, the epidote and allanite are dramatically zoned with respect to REEs, for example varying from $<1-5 \mathrm{wt}$ percentage total REE oxides in a single grain. No consistent zonation pattern was discovered. Within an individual sample, some grains are zoned with REE-rich cores to REE-poor rims while other display the opposite zonation (Table 7; Fig. 4b). In other grains the zonation in REEs does not vary consistently or smoothly from core to rim. The Sr contents also vary, from 1.5 to $4.0 \mathrm{wt} \% \mathrm{SrO}$ in epidote from the Liavatn marbles and from 0.3 to $1.1 \mathrm{wt} \% \mathrm{SrO}$ in allanite from the
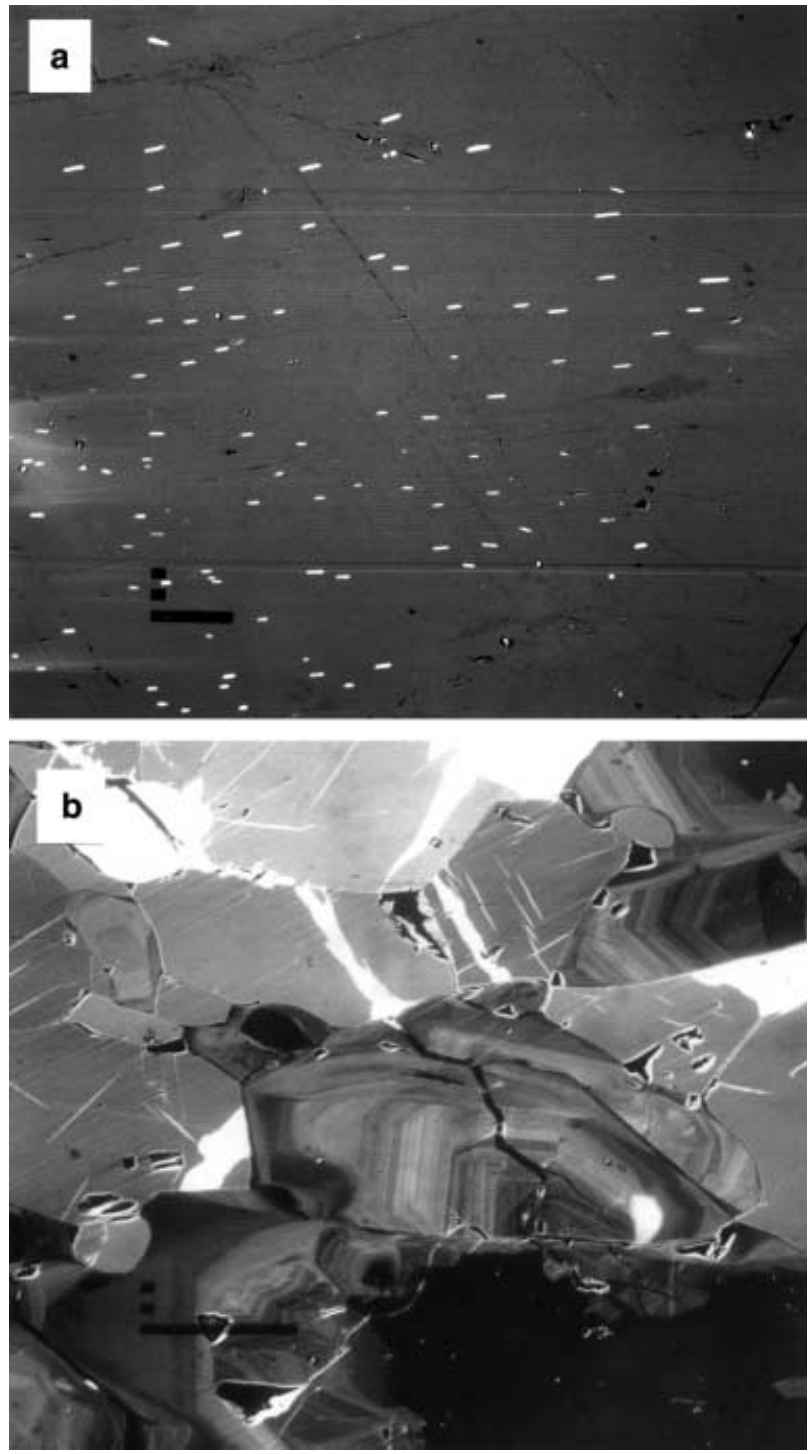

Fig. 4. Back-scattered electron microscope (BSE) images of samples used in the study. (a) Calcite (grey) with aligned inclusions of celestine (bright elongate needles) (from sample BA25/92 A, Sjurdholmen locality). The two lighter gray square patches in the calcite are the result of beam damage during microprobe analysis. The scale bar is $100 \mu \mathrm{m}$ (b) Zoning in allanite from sample BA69/93, Sjurdholmen locality. Lighter areas correspond to more REE enrichment, whereas the darkest areas of the allanite are the least REE enriched. The zoning shows several sequences of REE enrichment during growth from core to rim. The scale bar is $100 \mu \mathrm{m}$.

Sjurdholmen marbles. Zoisite crystals from the eclogite shear zones have similar Sr contents and have up to 2 wt.\% REE oxides (Boundy et al., 1992). One of the eclogite samples from the Sjurdholmen locality (BA69/93) has allanite similar to the adjacent marbles.

\section{Titanite}

The titanite analysed from the marbles is typically unzoned (Table 8). It contains variable amounts of 


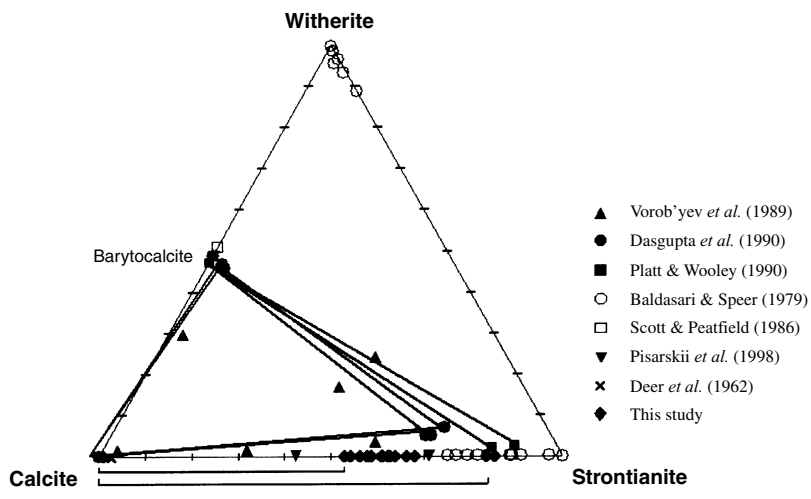

Fig. 5. $\mathrm{CaCO}_{3}-\mathrm{BaCO}_{3}-\mathrm{SrCO}_{3}$ ternary plot of orthorhombic carbonates from sample BA9/91 A demonstrating the extent of solid solution along the $\mathrm{CaCO}_{3}-\mathrm{SrCO}_{3}$ binary. Tie lines have been drawn between coexisting compositions. Filled circles represent carbonates from the Sausar group, India, metamorphosed at $660^{\circ} \mathrm{C}$ and 6 kbar (Dasgupta et al., 1990). Filled, inverted triangles represent travertine deposit analyses from Pisarskii et al. (1998).

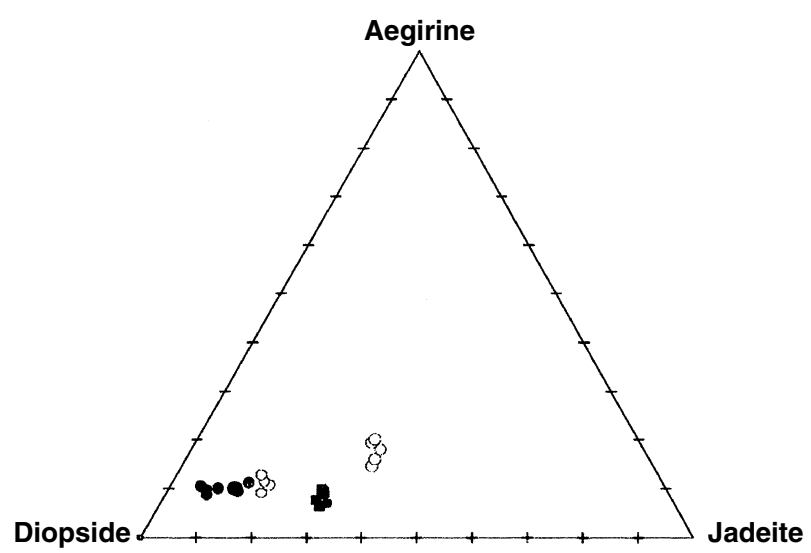

Fig. 6. Clinopyroxene compositions. Marble samples are shown as solid symbols and eclogite samples are shown as open symbols. The marble and eclogite samples from the Liavatn locality $(\boldsymbol{\square}, \square)$ are more jadeite rich than most of the Sjurdholmen locality $(\mathbf{\bullet}, \bigcirc)$ samples.

$\mathrm{Al}_{2} \mathrm{O}_{3}$ (1-3 wt.\%), with low contents of $\mathrm{F}(0.3$ wt. \%) and $\operatorname{REE}(<1$ wt. $\%)$.

\section{Celestine and barite}

The marble assemblages include celestine and a few have coexisting celestine and barite (Table 9). These are plotted in Fig. 8 and show two populations of barite and barite-celestine with solid solution of minor $\mathrm{CaSO}_{4}$. One sample (BA9/91 A) contains numerous clusters of barite $\left(\mathrm{Ba}_{0.49} \mathrm{Ca}_{0.01} \mathrm{Sr}_{0.50} \mathrm{SO}_{4}\right)$ and celestine $\left(\mathrm{Sr}_{0.96} \mathrm{Ba}_{0.04} \mathrm{SO}_{4}\right)$ in contact with one another (Fig. 9). A sharp grain boundary between the two phases is suggestive of a possible miscibility gap. Prieto et al. (1997) concluded that intermediate compositions of $(\mathrm{Ba}, \mathrm{Sr}) \mathrm{SO}_{4}$ are rare because of the extremely low

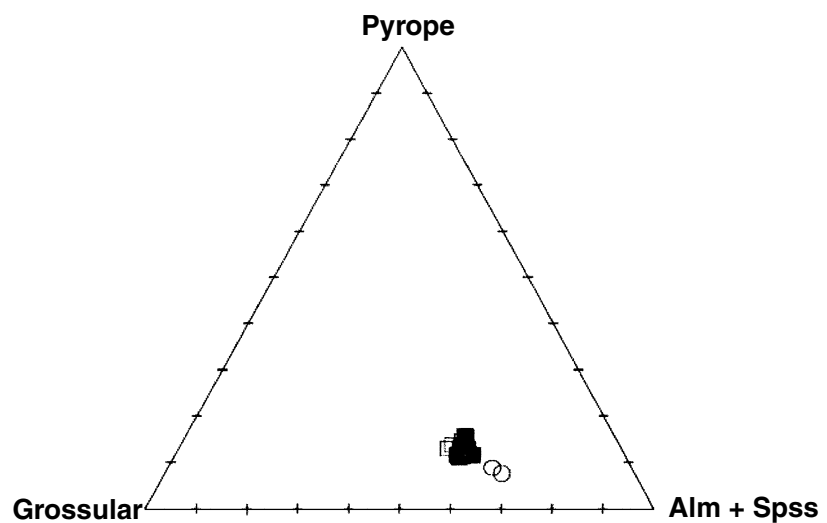

Fig. 7. Garnet compositions plotted as pyrope - grossular almandine + spessartine. Marble samples are shown as solid symbols and eclogite samples are shown as open symbols. Liavatn locality $(\boldsymbol{\square}, \square)$ and Sjurdholmen locality $(\mathbf{0}, \bigcirc)$ samples.

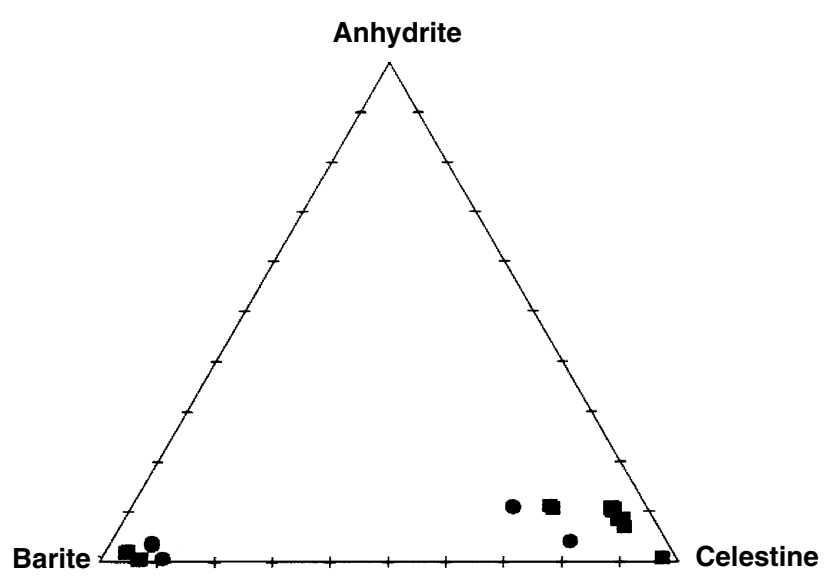

Fig. 8. Sulphate mineral compositions plotted as baritecelestine-anhydrite. Liavatn locality $(\mathbf{\square}, \square)$ and Sjurdholmen locality $(\bullet, \bigcirc)$ samples.

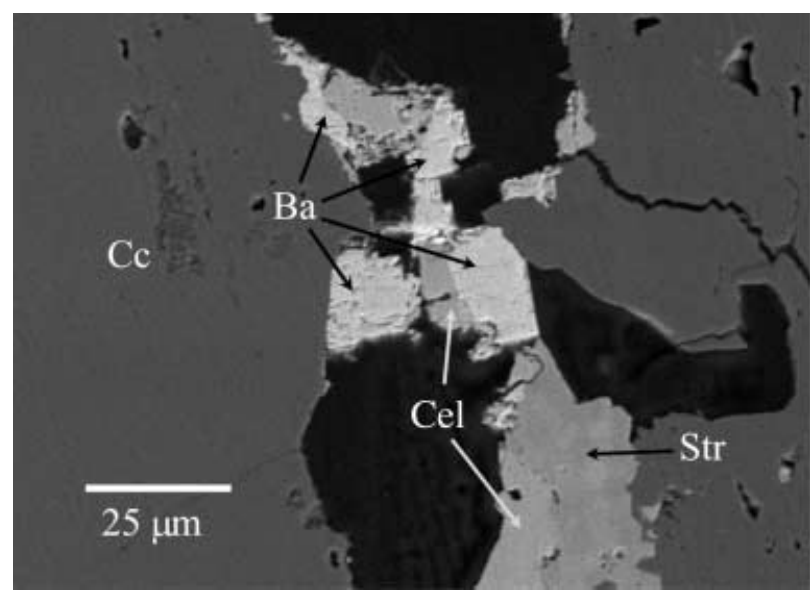

Fig. 9. BSE image of celestine $(\mathrm{Cel})$ and barite $(\mathrm{Ba})$, and strontianite (Str) in a matrix of calcite $(\mathrm{Cc})$ from marble sample BA9/91 A. The fairly sharp boundary is consistent with the presence of a miscibility gap between celestine and barite. 
solubility of $\mathrm{BaSO}_{4}$ relative to $\mathrm{SrSO}_{4}$, which leads to bimodal nucleation of these phases when precipitated from a fluid and that intermediate compositions are therefore only seen if the reacting composition is within a very narrow range. During metamorphic crystallization one would, however, expect to find a single composition, not a wide range in compositions for a single rock in the absence of a miscibility gap. In this study, the analytical data are rather scattered (Fig. 8). Whether a miscibility gap could extend to eclogitic $P-T$ conditions, or whether some of the sulphate could have been a high pressure polymorph (Liu \& Bassett, 1986), is difficult to evaluate without direct experiments.

\section{Biotite}

Biotite, analysed for one marble sample, BA25/92 A (Table 10), shows typical metamorphic compositions, with moderate $\mathrm{Ti}(c .2 \mathrm{wt} \%), X_{\mathrm{Ann}}=0.56$ and $X_{\mathrm{Phl}}=0.44$. Biotite has very low $\mathrm{Cl}$ and $\mathrm{F}$, typically $0.07 \pm 0.01 \mathrm{wt} \% \mathrm{Cl}$ and $0.01-0.08 \pm 0.05 \mathrm{wt} \% \mathrm{~F}$. This corresponds to only small amounts of halogens in the hydroxyl site (0.01 mol percentage).

\section{WHOLE ROCK CHEMISTRY}

The whole rock chemistry has been determined for two marble samples from Liavatn (Table 11). Given the high $\mathrm{Sr}$ content of the calcite and the presence of other phases, such as celestine, it is not surprising that the marbles have high bulk-rock $\mathrm{Sr}$ contents $(9,500$ 11000 p.p.m.; Table 11). The high $\mathrm{Sr}$ and Y contents are suggestive of a possible magmatic origin (Woolley \& Kempe, 1989), although Sr enrichment is also possible in some sedimentary environments as well. However, low concentrations of some other key trace elements, for example $\mathrm{Ba}$ (110-160 p.p.m.) and $\mathrm{Nb}$ ( $<5$ p.p.m.), suggests that the calc-silicate marble is not likely of magmatic origin (a former carbonatite). Since there is no independent evidence for a protolith from the field data, it is difficult to assess the extent of possible metasomatism during the cycling through the orogenic events. The surrounding granulite and eclogites of anorthositic to gabbroic compositions yield $\mathrm{Sr}$ contents of $c .1000$ p.p.m., Ba contents of $c .500$ p.p.m. (Rockow et al., 1997). An eclogite from Sjurdholmen (mangerite protolith?) reveals $\mathrm{Sr}$ contents of c. 698 p.p.m and unusually high Ce (3078 p.p.m.), Zr (4122 p.p.m.) Y (502 p.p.m.) and Nb (108 p.p.m.) contents (Svensen et al., 2001).

\section{Sr isotopes}

The ${ }^{87} \mathrm{Sr} /{ }^{86} \mathrm{Sr}$ ratios of four of the marble samples were determined at the University of Muenster. Samples from the Liavatn area have variable ${ }^{87} \mathrm{Sr} /{ }^{86} \mathrm{Sr}$ ratios, ranging from 0.7051 to 0.7059 (Table 12). The sample analysed from the Sjurdholmen locality yielded a ${ }^{87} \mathrm{Sr} /{ }^{86} \mathrm{Sr}$ ratio of 0.7058 . These ${ }^{87} \mathrm{Sr} /{ }^{86} \mathrm{Sr}$ values are
Table 11. XRF whole rock analyses.

\begin{tabular}{|c|c|c|}
\hline Sample no: & $\begin{array}{c}\text { Liavatn } \\
\text { HA4/90 A }\end{array}$ & $\begin{array}{l}\text { Liavatn } \\
\text { BA9/91 A }\end{array}$ \\
\hline \multicolumn{3}{|c|}{ Major elements (wt.\%) } \\
\hline $\mathrm{SiO}_{2}$ & 0.95 & 0.85 \\
\hline $\mathrm{TiO}_{2}$ & 0.04 & 0.02 \\
\hline $\mathrm{Al}_{2} \mathrm{O}_{3}$ & 0.69 & 0.69 \\
\hline $\mathrm{Fe}_{2} \mathrm{O}_{3}$ & 1.91 & 2.22 \\
\hline $\mathrm{MnO}$ & 0.20 & 0.19 \\
\hline $\mathrm{MgO}$ & 1.24 & 1.58 \\
\hline $\mathrm{CaO}$ & 51.21 & 51.12 \\
\hline $\mathrm{Na}_{2} \mathrm{O}$ & 0.00 & 0.00 \\
\hline $\mathrm{K}_{2} \mathrm{O}$ & 0.00 & 0.00 \\
\hline $\mathrm{P}_{2} \mathrm{O}_{5}$ & 1.71 & 0.10 \\
\hline $\mathrm{H}_{2} \mathrm{O}$ & 1.23 & 0.65 \\
\hline $\mathrm{CO}_{2}$ & 38.84 & 41.20 \\
\hline $\mathrm{Cr}_{2} \mathrm{O}_{3}$ & 0.00 & 0.00 \\
\hline $\mathrm{NiO}$ & 0.00 & 0.00 \\
\hline Total & 98.02 & 98.62 \\
\hline \multicolumn{3}{|c|}{ Trace elements (p.p.m.) } \\
\hline $\mathrm{Ba}$ & 161 & 112 \\
\hline $\mathrm{Rb}$ & 13 & 11 \\
\hline $\mathrm{Sr}$ & 11082 & 9533 \\
\hline $\mathrm{Pb}$ & 25 & 22 \\
\hline Th & 13 & 12 \\
\hline $\mathrm{U}$ & 42 & 35 \\
\hline $\mathrm{Nb}$ & $<5$ & $<5$ \\
\hline $\mathrm{La}$ & 63 & 46 \\
\hline $\mathrm{Ce}$ & 138 & 101 \\
\hline $\mathrm{Nd}$ & 87 & 70 \\
\hline Y & 130 & 115 \\
\hline $\mathrm{Zr}$ & 33 & 28 \\
\hline V & $<3$ & $<3$ \\
\hline $\mathrm{Cr}$ & $<3$ & $<3$ \\
\hline $\mathrm{Ni}$ & $<2$ & $<2$ \\
\hline Co & 14 & 11 \\
\hline $\mathrm{Cu}$ & 24 & 20 \\
\hline $\mathrm{Zn}$ & $<3$ & $<3$ \\
\hline $\mathrm{Ga}$ & $<1$ & $<1$ \\
\hline $\mathrm{Hf}$ & $<2$ & $<2$ \\
\hline S & 936 & 696 \\
\hline
\end{tabular}

Table 12. Isotopic analyses.

\begin{tabular}{llllr}
\hline Sample & Locality & Locality & $87 \mathrm{Sr} / 86 \mathrm{Sr}$ & Error \\
\hline HA4 90/4a & marble & Liavatn & 0.705256 & 9 \\
HA4 90/4b & marble & Liavatn & 0.705134 & 10 \\
HA5 90 & marble & Liavatn & 0.705860 & 10 \\
BA9/91 & marble & Liavatn & 0.705079 & 10 \\
BA25 92-4 & marble & Sjurdholmen & 0.705816 & 8 \\
\hline
\end{tabular}

more characteristic of crustal than mantle materials. The variability of ratios, even between samples from the same general locality, likely indicates that they have been modified from their original values or were heterogeneous from the beginning. Metamorphism, the influx of fluids, and metasomatism could variably alter the ${ }^{87} \mathrm{Sr} /{ }^{86} \mathrm{Sr}$ ratios. The marbles may well have experienced more than one phase of metamorphism and retrogression (Boundy, 1995; van Wyck et al., 1996). Unfortunately, the nature and extent of metasomatism in altering the isotopic ratios is not known. McCaig et al. (2000) documented infiltration of fluids rich in $\mathrm{Sr}$ can significantly effect the ${ }^{87} \mathrm{Sr} /{ }^{86} \mathrm{Sr}$ composition of carbonate rocks. Based on the field relations, whole rock chemistry and isotopic ratios, the precursor to the 
marbles remains unresolved at this time although the $\mathrm{Sr}$ isotopes are strongly suggestive of a crustal source (or modified crustal material).

\section{P-T-X CONDITIONS OF MARBLE METAMORPHISM}

\section{Thermobarometry}

Eclogites from Holsnøy Island (Lindås Nappe) are estimated to have formed at about $650-700{ }^{\circ} \mathrm{C}$ at pressures of 15-21 kbar (Austrheim \& Griffin, 1985; Jamtveit et al., 1990; Boundy et al., 1992). However, heretofore the equilibrium $P-T$ conditions of the marbles have not been evaluated. The calcite-dolomite thermometer of Anovitz \& Essene (1987) was used to place lower boundary constraints on the metamorphic temperature conditions experienced by the marbles. Since the $\mathrm{Mg}$ content of the calcite is commonly reduced by retrograde exsolution of dolomite and/or recrystallization, temperature estimates based on the calcite-dolomite solvus often represent a minimum temperature of formation (Essene, 1983). Furthermore, in the absence of dolomite, the calcite compositions can only be used to estimate minimum temperatures. Calcite and dolomite were found together in only one sample in this study (BA9/91 A, Liavatn locality). However, the texture is suggestive of exsolution, and therefore the coexistence of the two phases under equilibrium conditions cannot be assumed. Temperature estimates using the calcite-dolomite solvus are consistent within a given sample. No discernible intra or intercrystalline variation within a given sample was found (Table 2). For the Liavatn marble, the temperature estimates range from 460 to $490{ }^{\circ} \mathrm{C}$, with one sample recording about $390{ }^{\circ} \mathrm{C}$ (Table 1). One marble from the Sjurdholmen locality yielded estimates of about $420^{\circ} \mathrm{C}$.

Temperature estimates were obtained from intercalated calc-silicate rocks and adjacent eclogite samples using the garnet-clinopyroxene thermometer of Krogh Ravna (2000) and assuming pressures of 20 kbar. The temperatures calculated using Krogh Ravna (2000) are typically lower than those estimated using the thermometer of Krogh (1988; Table 13). For the eclogite sample from Liavatn, garnet and clinopyroxene yield temperatures of about 640 $690{ }^{\circ} \mathrm{C}$, the calc-silicate garnet with included omphacite from Liavatn $580-650{ }^{\circ} \mathrm{C}$, and the eclogite sample from the Sjurdholmen locality about $595^{\circ} \mathrm{C}$ (Table 13). Previous temperature estimates for the Holsnøy eclogites range from 650 to $700{ }^{\circ} \mathrm{C}$ (Austrheim \& Griffin, 1985; Jamtveit et al., 1990; Boundy et al., 1992). The lower temperature estimates from marbles and calc-silicates and the Sjurdholmen eclogite may indicate re-equilibration at lower temperatures during retrogression.

The presence of omphacite with jadeite contents of up to $30 \%$ implies that marble formed under high
Table 13. Garnet - pyroxene thermometry of eclogites and calc-silicates.

\begin{tabular}{|c|c|c|c|c|c|c|}
\hline Sample & grt & $\operatorname{cpx}$ & $\mathrm{K}_{\mathrm{D}}$ & $T^{\circ} \mathrm{C}(\mathrm{K} 88)$ & $T^{\circ} \mathrm{C}(\mathrm{K} 00)$ & $\begin{array}{c}(\mathrm{K} 00-\mathrm{K} 88) \\
\Delta T\end{array}$ \\
\hline \multicolumn{7}{|c|}{ Liavatn eclogite sample } \\
\hline Liavatn & $1 / 3 \mathrm{r}$ & $2 / 2 \mathrm{r}$ & 10.9 & 671 & 644 & -27 \\
\hline Liavatn & $1 / 4 \mathrm{r}$ & $1 / 2 \mathrm{r}$ & 10.5 & 687 & 663 & -24 \\
\hline Liavatn & $2 / 2 \mathrm{r}$ & $4 / 2 \mathrm{r}$ & 11.6 & 669 & 640 & -29 \\
\hline Liavatn & $3 / 2 \mathrm{r}$ & $4 / 3 \mathrm{r}$ & 9.3 & 721 & 693 & -28 \\
\hline Liavatn & $4 / 3 \mathrm{r}$ & $3 / 2 \mathrm{r}$ & 11.9 & 671 & 639 & -32 \\
\hline Averages: & & & 10.8 & 684 & 656 & -28 \\
\hline \multicolumn{7}{|c|}{ Sjurdholmen eclogite sample } \\
\hline BA $69 / 93$ & $1 / 1 \mathrm{c}$ & $2 / 1 \mathrm{c}$ & 13.3 & 586 & 597 & 11 \\
\hline \multirow[t]{2}{*}{ BA69/93 } & $1 / 2 \mathrm{r}$ & $2 / 4 \mathrm{r}$ & 14.3 & 583 & 592 & 8 \\
\hline & & & 13.8 & 585 & 594 & 10 \\
\hline \multicolumn{7}{|c|}{ Liavatn calc-silicate sample - garnets with cpx inclusions } \\
\hline $\mathrm{BA} 21 / 93 \mathrm{c}$ & 9 & 3 & 13.1 & 628 & 586 & -42 \\
\hline BA $21 / 93 \mathrm{c}$ & 2 & 9 & 12.2 & 629 & 592 & -37 \\
\hline BA21/93 c & 5 & 12 & 13.8 & 621 & 577 & -44 \\
\hline BA $21 / 93 \mathrm{c}$ & 4 & 7 & 9.5 & 688 & 650 & -38 \\
\hline Averages: & & & 12.2 & 642 & 602 & -40 \\
\hline
\end{tabular}

K88=Krogh (1988)@20 kbar

$\mathrm{K} 00=\mathrm{Krogh}$ Ravna (2000) @20 kbar

$r=\operatorname{rim} ; \mathrm{c}=$ core.

pressure conditions. However, in the absence of both quartz and albite, the jadeite content is not useful in constraining peak pressures (Holland, 1980). The adjacent eclogite sample from the Liavatn locality also has omphacite with $\mathrm{Jd}_{30}$, and the assemblage includes coexisting quartz. Therefore, using the reaction

$$
\begin{array}{ccc}
\mathrm{NaAlSi}_{3} \mathrm{O}_{8}= & \mathrm{NaAlSi}_{2} \mathrm{O}_{6} & +\mathrm{SiO}_{2} \\
\text { al } & \mathrm{Jd} \text { in } & \text { Qtz } \\
& \text { omp } &
\end{array}
$$

at the estimated average temperature of $660{ }^{\circ} \mathrm{C}$ yields a minimum pressure of about $13 \mathrm{kbar}$ in the absence of albite (Holland, 1980). The field association with eclogites and the jadeite content of clinopyroxene from the marbles strongly suggests that the marbles experienced eclogite facies conditions.

\section{Fluid composition}

Fluid compositions associated with high-pressure metamorphism of the marbles from Holsnøy Island are of interest in order to compare with those from the eclogites. The activity of fluid components in a mixed $\mathrm{CO}_{2}-\mathrm{H}_{2} \mathrm{O}$ fluid can vary dramatically in highpressure rocks. Austrheim (1990) illustrated variable activities of components of infiltrating fluids during the eclogitization of granulite facies ultramafites from Holsnøy Island. Svensen et al. (2001) showed a mafic eclogite from Sjurdholmen may have equilibrated with $\mathrm{H}_{2} \mathrm{O}$-rich fluids containing high levels of halogens. At elevated pressures, dissociated salts can dramatically reduce $a \mathrm{H}_{2} \mathrm{O}$ and increase $\mathrm{SiO}_{2}$ solubility (Shmulovitch \& Graham, 1999). Thus, further evaluation of the fluids associated with the marbles and the surrounding eclogites is critical in evaluating the phase equilibria. 
The halogen component of the fluid has been investigated by undertaking $\mathrm{Cl}$ and $\mathrm{F}$ analysis of biotite on the electron microprobe, giving averages of $0.07 \pm 0.01 \mathrm{wt}$ percentage for $\mathrm{Cl}$ and $0.01 \pm 0.05 \mathrm{wt}$ percentage for $\mathrm{F}$ Table (10). These values are less than a third of those reported by Svensen et al. (2001) for Cl and one tenth of those for $\mathrm{F}$ in biotite with the highest halogen content from WGR eclogite samples. However, halogen contents of hydrous silicates in eclogites from Sjurdholmen area are below the EMP detection limit (Svensen et al., 2001). Therefore the increased halogens in the Sjurdholmen area were only evidenced locally in the evolved fluid inclusions. This suggests higher $a \mathrm{H}_{2} \mathrm{O}$ since $\mathrm{Cl}$ prefers to remain in solution and not enter into hydrous minerals under high $a \mathrm{H}_{2} \mathrm{O}$. Using the experiments of Munoz \& Swenson (1981) and the recalibration of Munoz (1992), the fluid in equilibrium with the biotite is estimated to have $f \mathrm{HCl}<0.021$. This value is an upper limit assuming $f \mathrm{H}_{2} \mathrm{O}=$ unity, but nonetheless gives a maximum $f \mathrm{HCl}$. The $\log \mathrm{K}$ of the following reaction yields an upper limit for the activity of $\mathrm{NaCl}$ :

$$
\begin{aligned}
& 2 \mathrm{HCl}+2 \mathrm{NaAlSi}_{2} \mathrm{O}_{6} \\
& \quad=\mathrm{Al}_{2} \mathrm{SiO}_{5}+3 \mathrm{SiO}_{2}+2 \mathrm{NaCl}+\mathrm{H}_{2} \mathrm{O}
\end{aligned}
$$

This reaction gives a maximum activity of $\mathrm{NaCl}=$ 0.019 at $600{ }^{\circ} \mathrm{C}$ and $20 \mathrm{kbar}$ in the absence of kyanite and quartz and using the above $\mathrm{HCl}, \mathrm{H}_{2} \mathrm{O}$, and jadeite components. The mole fraction of $\mathrm{NaCl}$ has been calculated using the $a / X$ relations experimentally determined by Aranovich \& Newton (1996) and yields values of $X_{\mathrm{NaCl}}=0.023$. This halogen component in the fluid is much lower than that of the brines suggested by Svensen et al. (2001) in other Norwegian eclogites.

The effect of dissolved $\mathrm{SiO}_{2}$ on $a \mathrm{H}_{2} \mathrm{O}$ in the peak metamorphic fluid may be estimated from the experiments of Manning (1994), who found 2.87 wt. $\% \mathrm{SiO}_{2}$ $\left(X \mathrm{SiO}_{2}=0.009\right)$ dissolved in $\mathrm{H}_{2} \mathrm{O}$ at $600{ }^{\circ} \mathrm{C}$ and $20 \mathrm{kbar}$ in the presence of quartz. Using Raoult's law $\left(a \mathrm{H}_{2} \mathrm{O}=X \mathrm{H}_{2} \mathrm{O}\right)$ for small amounts of dilution leads to a reduction in the activity of $\mathrm{H}_{2} \mathrm{O}$ by a factor of only 0.991 . This provides a maximum estimate of dilution by $\mathrm{SiO}_{2}$ for the Holsnøy marbles, which lack quartz. In the absence of an excess fluid phase, the $T-X$ equilibria generally move to lower temperature with decreasing fluid pressures but will not strongly affect the inferences of a fictive fluid composition. The presence of hydrous minerals such as biotite in the marble severely limits any reduction in fluid pressure (Edwards \& Essene, 1988). The use of $T /\left(X \mathrm{H}_{2} \mathrm{O}+X \mathrm{CO}_{2}\right)$ diagrams is an excellent approximation for these rocks, which have low salinities as recorded by their $\mathrm{Cl}$ contents and only minor solution of $\mathrm{SiO}_{2}$ in the fluid phase.

The $f \mathrm{O}_{2}$ was calculated for the garnet-bearing calc-silicate sample (BA21/93) using the method of Donohue \& Essene (2000), which utilizes coexisting garnet and epidote via the reaction:

$$
\begin{aligned}
2 \mathrm{Ca}_{2} \mathrm{FeAl}_{2} \mathrm{Si}_{3} \mathrm{O}_{12}(\mathrm{OH})= & 2 \mathrm{Ca}_{2} \mathrm{FeAl}_{2} \mathrm{Si}_{3} \mathrm{O}_{12} \\
& +\mathrm{H}_{2} \mathrm{O}+1 / 2 \mathrm{O}_{2} \\
\mathrm{Ep}\left(\mathrm{Ps}_{33}\right)= & \mathrm{Grt}
\end{aligned}
$$

The results indicate fairly oxidizing conditions during metamorphism, approximately $0.3-0.5 \log f \mathrm{O}_{2}$ units above the hematite-magnetite buffer over the calculated range of $400-700{ }^{\circ} \mathrm{C}$ at a fixed pressure of $18 \mathrm{kbar}$ (Fig. 10). These conditions would not permit a significant $\mathrm{CH}_{4}$ component in the fluid.

We suggest that an $\mathrm{H}_{2} \mathrm{O}-\mathrm{CO}_{2}$ binary is a good approximation for the composition of the fluid for the Holsnøy marbles. For samples with coexisting quartz, upper limits on $X_{\mathrm{CO} 2}$ can be calculated at the estimated $P-T$ using reactions like:

$$
\begin{aligned}
\mathrm{CaMg}\left(\mathrm{CO}_{3}\right)_{2}+2 \mathrm{SiO}_{2} & =\mathrm{CaMgSi}_{2} \mathrm{O}_{6}+2 \mathrm{CO}_{2} \\
\mathrm{Dol}+\mathrm{Qtz} & =\mathrm{Di}(\text { in Omp })+\text { fluid } \\
\mathrm{CaCO}_{3}+\mathrm{TiO}_{2}+\mathrm{SiO}_{2} & =\mathrm{CaTiSiO}_{5}+\mathrm{CO}_{2} \\
\mathrm{Cc}+\mathrm{Ru}+\mathrm{Qtz} & =\mathrm{Ttn}+\text { fluid }
\end{aligned}
$$

Using reaction (4) for similar composition clinopyroxene from eclogite facies marbles from the Dabie Mountains in Central China, Wang \& Liou, 1993) calculated very low $X_{\mathrm{CO}_{2}}$ values $(<0.03)$. The Dabie marbles and eclogites are estimated to have formed

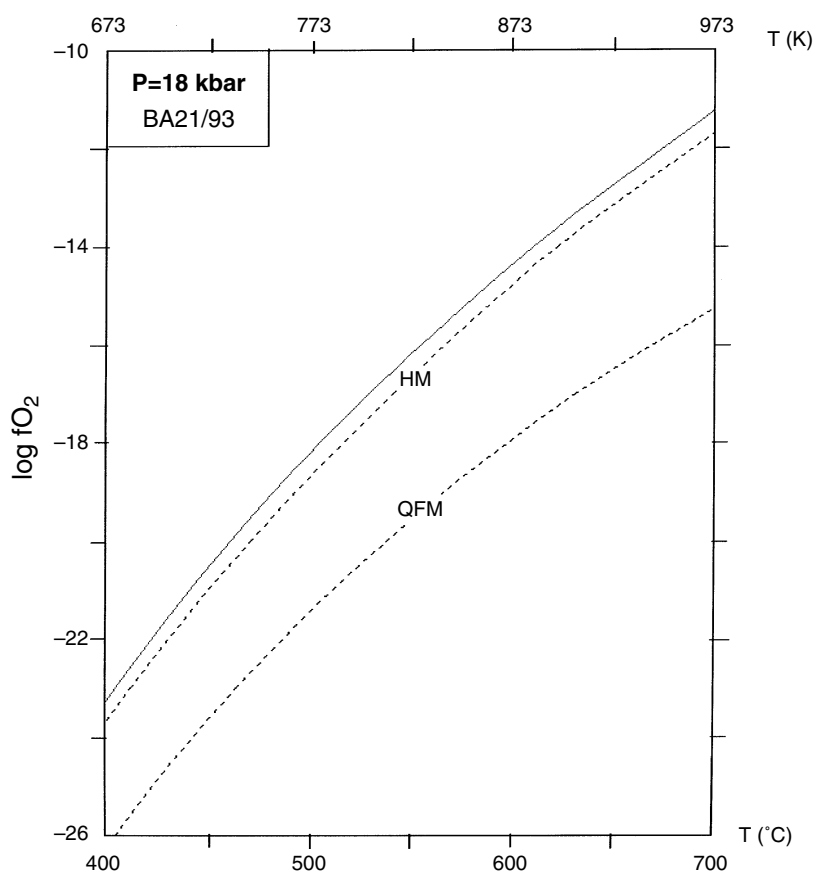

Fig. 10. $\log f \mathrm{O}_{2}-T$ diagram calculated for equation 14 and garnet - epidote compositions from sample BA21/93 (solid line). Shown as references are the solid oxygen buffer curves (dashed lines) for hematite - magnetite (HM) and quartz - fayalite magnetite (QFM). Pressure was fixed at $18 \mathrm{kbar}$ for this calculation. An uncertainty of \pm 5 kbar generates $\pm 1.0 \mathrm{log}$ units in $f \mathrm{O}_{2}$ (Donohue \& Essene, 2000). 

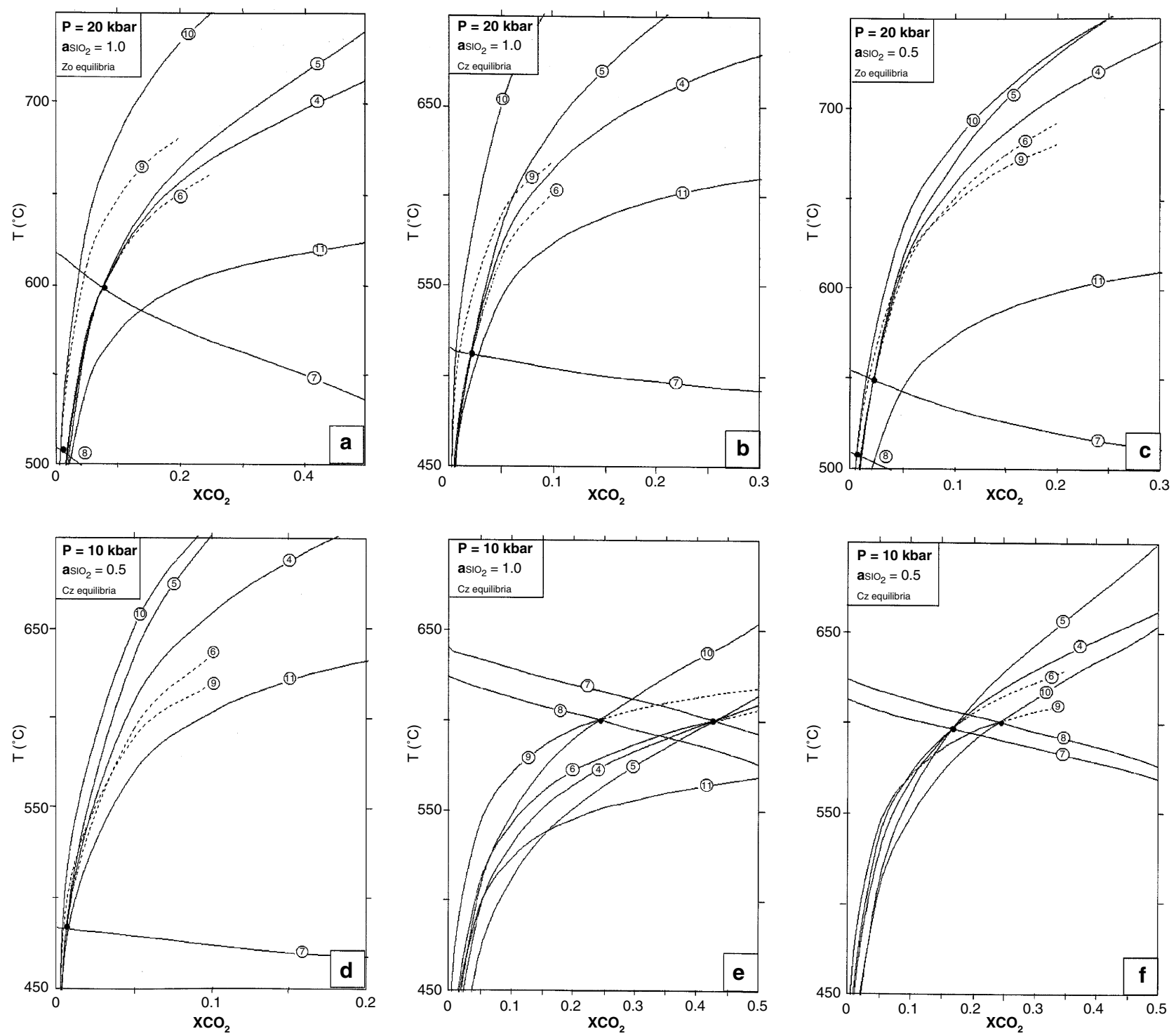

Fig. 11. (a-f) $T-X \mathrm{CO}_{2}$ diagrams for calc-silicate sample BA21/93. Solid lines represent stable reactions while dashed lines show metastable extensions. Diagrams 11a-d were calculated at $20 \mathrm{kbar}$ using zoisite (Zo) thermodynamic data for $11 \mathrm{a} \& 11 \mathrm{c}$ and clinozoisite $(\mathrm{Cz})$ data for $11 \mathrm{~b} \& 11 \mathrm{~d}$. Figure $11(\mathrm{c}, \mathrm{d})$ shows the effects of reduced silica activity on the locus of reactions in each system. Figure 11(e,f) shows the effects of reduced pressure on the equilibria of Fig. 11(b,d). The limiting reactions for zoisite (Zo), clinozoisite $(\mathrm{Cz})$, and grossular (Grs) are labelled with the appropriate abbreviations.

at similar temperatures to the Holsnøy eclogites, although at higher minimum pressures $(>25 \mathrm{kbar})$ based on the occurrence of coesite.

$T-X_{\mathrm{CO}}$ equilibria have been constructed for one calc-silicate sample, BA21/93 (Fig. 11a-f) using the following dehydration and decarbonation reactions corrected for solid solutions:

$$
\begin{aligned}
\mathrm{Ca}_{3} \mathrm{Al}_{2} \mathrm{Si}_{3} \mathrm{O}_{12}+3 \mathrm{CO}_{2}= & 3 \mathrm{CaCO}_{3}+\mathrm{Al}_{2} \mathrm{SiO}_{5} \\
& +2 \mathrm{SiO}_{2} \\
\text { Grs }+ \text { fluid }= & \mathrm{Cc}+\mathrm{Ky}+\mathrm{Qtz}
\end{aligned}
$$

$$
\begin{gathered}
2 \mathrm{Ca}_{2} \mathrm{Al}_{3} \mathrm{Si}_{3} \mathrm{O}_{12}(\mathrm{OH})+4 \mathrm{CO}_{2} \\
=4 \mathrm{CaCO}_{3}+3 \mathrm{Al}_{2} \mathrm{SiO}_{5}+3 \mathrm{SiO}_{2}+\mathrm{H}_{2} \mathrm{O} \\
\mathrm{Czo}+\text { fluid }=\mathrm{Cc}+\mathrm{Ky}+\mathrm{Qtz}+\mathrm{H}_{2} \mathrm{O} \\
2 \mathrm{Ca}_{2} \mathrm{Al}_{3} \mathrm{Si}_{3} \mathrm{O}_{12}(\mathrm{OH})+5 \mathrm{CaCO}_{3}+3 \mathrm{SiO}_{2} \\
=3 \mathrm{Ca}_{3} \mathrm{Al}_{2} \mathrm{Si}_{3} \mathrm{O}_{12}+\mathrm{H}_{2} \mathrm{O}+5 \mathrm{CO}_{2} \\
\mathrm{Czo}+\mathrm{Cc}+\mathrm{Qtz}=\mathrm{Grs}+\mathrm{H}_{2} \mathrm{O}+\text { fluid } \\
6 \mathrm{Ca}_{2} \mathrm{Al}_{3} \mathrm{Si}_{3} \mathrm{O}_{12}(\mathrm{OH})=4 \mathrm{Ca}_{3} \mathrm{Al}_{2} \mathrm{Si}_{3} \mathrm{O}_{12} \\
+5 \mathrm{Al}_{2} \mathrm{SiO}_{5}+3 \mathrm{SiO}_{2}+\mathrm{H}_{2} \mathrm{O} \\
\mathrm{Czo}=\mathrm{Grs}+\mathrm{Ky}+\mathrm{Qtz}+\mathrm{H}_{2} \mathrm{O}
\end{gathered}
$$




$$
\begin{aligned}
& 6 \mathrm{Ca}_{2} \mathrm{Al}_{3} \mathrm{Si}_{3} \mathrm{O}_{12}(\mathrm{OH})+\mathrm{Al}_{2} \mathrm{O}_{3}=6 \mathrm{Al}_{2} \mathrm{SiO}_{5} \\
& +4 \mathrm{Ca}_{3} \mathrm{Al}_{2} \mathrm{Si}_{3} \mathrm{O}_{12}+3 \mathrm{H}_{2} \mathrm{O} \\
& \mathrm{Czo}+\mathrm{Crn}=\mathrm{Ky}+\mathrm{Grs}+\mathrm{H}_{2} \mathrm{O} \\
& 2 \mathrm{Ca}_{2} \mathrm{Al}_{3} \mathrm{Si}_{3} \mathrm{O}_{12}(\mathrm{OH})+2 \mathrm{CaCO}_{3} \\
& =2 \mathrm{Ca}_{3} \mathrm{Al}_{2} \mathrm{Si}_{3} \mathrm{O}_{12}+\mathrm{Al}_{2} \mathrm{O}_{3}+2 \mathrm{CO}_{2}+\mathrm{H}_{2} \mathrm{O} \\
& \mathrm{Czo}+\mathrm{Cc}=\mathrm{Grs}+\mathrm{Crn}+\text { fluid }+\mathrm{H}_{2} \mathrm{O} \\
& 2 \mathrm{Ca}_{2} \mathrm{Al}_{3} \mathrm{Si}_{3} \mathrm{O}_{12}(\mathrm{OH})+3 \mathrm{Al}_{2} \mathrm{O}_{3}+4 \mathrm{CO}_{2} \\
& =6 \mathrm{Al}_{2} \mathrm{SiO}_{5}+4 \mathrm{CaCO}_{3}+\mathrm{H}_{2} \mathrm{O} \\
& \mathrm{Czo}+\mathrm{Crn}+\text { fluid }=\mathrm{Ky}+\mathrm{Cc}+\mathrm{H}_{2} \mathrm{O} \\
& \mathrm{Ca}_{2} \mathrm{Mg}_{5} \mathrm{Si}_{8} \mathrm{O}_{22}(\mathrm{OH})_{2}+3 \mathrm{CaCO}_{3}+2 \mathrm{SiO}_{2} \\
& =5 \mathrm{CaMgSi}_{2} \mathrm{O}_{6}+3 \mathrm{CO}_{2}+\mathrm{H}_{2} \mathrm{O} \\
& \mathrm{Tr}+\mathrm{Cc}+\mathrm{Qtz}=\mathrm{Di}+\text { fluid }+\mathrm{H}_{2} \mathrm{O}
\end{aligned}
$$

All $T-X_{\mathrm{CO}_{2}}$ equilibria were calculated using THERMOCALC (Holland \& Powell, 1998) and the equation of state for the fluid was taken directly from this data set. Reactions involving epidote were calculated for both clinozoisite and zoisite because the crystal structure present in the sample is unknown and the thermodynamic properties for the two species are significantly different (e.g. Robie \& Hemingway, 1995; Holland \& Powell, 1998). Figure 11(a,b) shows the $T-X_{\mathrm{CO}_{2}}$ relations for sample BA21/93 at $20 \mathrm{kbar}$ and an activity of silica $\left(a_{\mathrm{SiO}_{2}}\right)=1$. Reactions $6,7,8$ and 9 form an invariant point involving the phases (clino)zoisite-grossular-calcite-kyanite-quartz- $\mathrm{H}_{2} \mathrm{O}-$

$\mathrm{CO}_{2}$. Reactions 10, 11, and 12 (the corundum analogues of reactions $7,8,9$ ) also form an invariant point at lower temperature and lower $X_{\mathrm{CO}_{2}}$. Reactions 7 and 9 limit the epidote stability in the absence of kyanite. Reaction 6 is the lower stability for grossular. The reaction space for BA21/93 is therefore limited to the region below reaction 9 and left of reaction 8 , although it is possible that the assemblage is located directly on the invariant point. This implies that at $20 \mathrm{kbar}$ and $a_{\mathrm{SiO}_{2}}=1$, the $X_{\mathrm{CO}_{2}}$ must be $<0.08$ for the zoisite equilibria or $<0.025$ for the clinozoisite equilibria regardless of the temperature estimate. If the $a_{\mathrm{SiO}_{2}}$ is reduced to 0.5 from 1.0 (Fig. 11c,d), the quartz-present invariant point for both the zoisite and clinozoisite reactions shifts to even more $\mathrm{H}_{2} \mathrm{O}$ rich conditions. Since quartz has not been found in sample BA21/93, it is possible that the $a_{\mathrm{SiO} 2}$ is indeed $<1.0$.

Lowering the pressure at which these calculations are performed has the effect of driving the loci of the invariant points towards much more $\mathrm{CO}_{2}$-rich conditions (Fig. 11e,f). While the pressure estimates of these rocks (18-21 kbar) seems reasonable based on the data for the associated eclogites, the equilibrium pressure of these rocks is not uniquely known. If the Liavatn marbles did equilibrate at significantly lower pressures than the adjacent eclogites, then the accompanying fluid would have been much more $\mathrm{CO}_{2}$ rich. However, the presence of abundant epidote in the marbles indicates high $X_{\mathrm{H}_{2} \mathrm{O}}$ conditions, implying that the marbles may have indeed equilibrated at high pressures.

If sufficient limits can be placed on the $X_{\mathrm{CO}_{2}}$ and $f\left(\mathrm{O}_{2}\right)$ the buffered equilibria

$$
\begin{gathered}
2 \mathrm{CaSO}_{4}+2 \mathrm{CO}_{2}=2 \mathrm{CaCO}_{3}+\mathrm{S}_{2}+3 \mathrm{O}_{2} \\
\text { Ahy }=\mathrm{Cc} \\
(\text { solid solution in barite or celestine) } \\
2 \mathrm{SrCO}_{3}+\mathrm{S}_{2}+3 \mathrm{O}_{2}=2 \mathrm{SrSO}_{4}+2 \mathrm{CO}_{2} \\
\mathrm{Str}=\mathrm{Cel}
\end{gathered}
$$

(solid solution in calcite)

could then provide upper limits on $\mathrm{SO}_{2}, \mathrm{SO}_{3}$, and sulphur fugacity $\left(f \mathrm{~S}_{2}\right)$, once the $a / \mathrm{X}$ relations of $\mathrm{Ca}-\mathrm{Ba}-\mathrm{Sr}$ sulphates and carbonates have been established.

\section{DISCUSSION}

\section{Conditions of metamorphism}

Marbles and calc-silicates from the BAS area subparallel Caledonian shear zones and appear to be thoroughly affected by the Caledonian deformation and metamorphism. Their intimate occurrence with eclogites and the jadeite content of the clinopyroxene indicates that the marbles experienced the same highpressure metamorphism. The marbles may even have formed from the precipitation from fluids generated during the high-pressure metamorphism and metasomatism of the granulites. For comparison, using the same thermobarometers employed by Boundy et al. (1992), the adjacent eclogite from the Liavatn locality yields temperatures of $670-720^{\circ} \mathrm{C}$ and minimum pressures of $13 \mathrm{kbar}$. These estimates are similar to the temperatures and pressures estimated in Boundy et al. (1992) $\left(T=670{ }^{\circ} \mathrm{C}, P_{\min }=15 \mathrm{kbar}\right)$ for eclogites in the NW Holsnøy Island area. The data from the Liavatn calc-silicate sample with garnet and omphacite yields temperatures of $580-650{ }^{\circ} \mathrm{C}$. These lower temperatures are consistent with those estimated from C isotopes, which are interpreted to represent retrograde temperatures of approximately $600{ }^{\circ} \mathrm{C}$ related to the influx of fluids (Van Wyck et al., 1996). Marbles, calcsilicates and some of the associated eclogites record lower temperatures than peak high-pressure eclogite facies temperatures in the area, which may indicate some retrogression to lower temperatures.

\section{Fluid evolution during high-pressure metamorphism}

Fluid released during subduction is an important catalyst for metamorphism, as documented in the BAS (e.g. Austrheim, 1987; Jamtveit et al., 1990). The 
chemistry of such fluids could be continuously modified by dehydration and hydration processes, as fluids are released and consumed during metamorphic reactions at depth (e.g. Svensen et al., 2001). Eclogite formation in the area resulted from the localized infiltration of fluids along fractures and shear zones (e.g. Austrheim. 1987). This may not have been a single pervasive invasion of a single fluid. From a study of a mafic eclogite from Sjurdholmen, Svensen et al. (1999) suggested that infiltrating $\mathrm{H}_{2} \mathrm{O}$-rich brines caused eclogitization and that their composition was modified during hydration. Phase equilibrium data from the carbonates support that the marbles and calc-silicates were in equilibrium with a $\mathrm{CO}_{2}$-poor fluid during eclogite facies metamorphism under relatively oxidizing conditions $\left(\mathrm{XCO}_{2}<0.08\right.$ at $\left.20 \mathrm{kbar}\right)$. The results presented here are consistent with previous investigations of phase equilibria of the eclogites from Holsnøy Island, which estimate $\mathrm{H}_{2} \mathrm{O}$ dominated fluid compositions ( $\geq 0.75$; Jamtveit et al., 1990). The fluids in equilibrium with the carbonates does not appear to be a (water-rich) brine, as indicated by Svensen et al. (1999), suggesting the variable nature of the fluids during high pressure eclogite metamorphism of the area. Different composition fluids may have infiltrated the area at depth and the fluid compositions evolved during high-pressure metamorphic reactions progressed. As hydrous fluids are consumed during highpressure eclogite metamorphism, the remaining evolved fluids could become more saline (Svensen et al., 1999).

Evaluation of similar marbles from other areas that experienced high-pressure metamorphism also suggests $\mathrm{H}_{2} \mathrm{O}$ dominated fluids (e.g. Franz \& Spear, 1983; Wang \& Liou, 1993). For example, in marbles of high pressure and ultrahigh pressure metamorphism from the Dabie Shan, the $X_{\mathrm{CO}}<0.03$ (Wang \& Liou, 1993) and $\mathrm{Su}-\mathrm{Lu}$ terrane the $X_{\mathrm{CO}_{2}}=0.001$ (Kato et al., 1997). High-pressure calc-silicates from Bavaria yield $X_{\mathrm{CO}_{2}} \leq 0.03$ (Klemd et al., 1994). Very low $X_{\mathrm{CO}_{2}}$ values have also been reported for high-pressure eclogite facies metamorphic rocks in the Alps (e.g. Holland, 1979; Franz \& Spear, 1983; Castelli, 1991). Explanations for the very low $X_{\mathrm{CO}_{2}}$ values may include: (1) lack of progress for decarbonation reactions during subduction to about $100 \mathrm{~km}$; (2) dominance of dehydration reactions producing substantial free $\mathrm{H}_{2} \mathrm{O}$, which are common during subduction-zone metamorphism (e.g. Peacock, 1992); and (3) almost complete fractionation of the $\mathrm{CO}_{2}$ component at high pressure into the solid phases (Molina \& Poli, 2000).

The degree of external vs. internal buffering of the fluid is variable depending upon fluid-rock ratios. Despite the influx of free fluids in the rocks at high pressure, the isotopic heterogeneities are best reconciled with local buffering and limited mobility of the fluids, at least during the final stage of equilibration of the Holsnøy eclogites (Boundy et al., 1997b). Limited fluid migration has also been suggested by Svensen et al. (2001). They interpreted multiphase brine inclusions in omphacite to reflect enrichment of elements incompatible with the silicate structure during consumption of $\mathrm{H}_{2} \mathrm{O}$ by eclogite-forming hydration reactions. Similarly, data from high-pressure eclogites from subduction zones supports limited fluid/rock ratios (Selverstone et al., 1992; Getty \& Selverstone, 1994). Because most dewatering and devolatilization during tectonic burial occurs prior to eclogite facies metamorphism, the volumetric fluid-rock ratio in eclogites should be generally low at subduction depths of $>45 \mathrm{~km}$ (Getty \& Selverstone, 1994).

For the Holsnøy Island localities, it is at present not known whether the $\mathrm{CO}_{2}$-poor fluid compositions reflect local buffering or a regional external source. Eclogites formed as a result of deformation and infiltration of fluids into relatively anhydrous Precambrian granulite facies rocks (e.g. Austrheim \& Griffin, 1985; Austrheim, 1987; Austrheim et al., 1997). This implies a fluid mobility on scales at least exceeding those of the precursors. The lack of nearby sources of extensive hydrous phases suggests the fluid source (for the initial retrogression) is not local. Breakdown of scapolite in the surrounding granulites during high-pressure eclogite facies metamorphism, however, would imply a local, limited $\mathrm{CO}_{2}$ source for the marbles, and could be controlled by reactions such as:

$$
\begin{aligned}
& 3 \mathrm{Ca}_{4} \mathrm{Al}_{6} \mathrm{Si}_{6} \mathrm{O}_{24} \mathrm{CO}_{3}=4 \mathrm{Ca}_{3} \mathrm{Al}_{2} \mathrm{Si}_{3} \mathrm{O}_{12} \\
& \quad+5 \mathrm{Al}_{2} \mathrm{SiO}_{5}+\mathrm{SiO}_{2}+3 \mathrm{CO}_{2} \\
& \text { meionite }=\mathrm{Grs}+\mathrm{Ky}+\mathrm{Qtz}+\text { fluid }
\end{aligned}
$$

(Moecher \& Essene, 1990).

\section{Origin of the marbles: metasomatic interactions with fluids?}

Currently a definitive origin of the marbles remains controversial, yet the data available suggest some intriguing possibilities. Marbles from the Liavatn area have calcite with average $\delta^{18} \mathrm{O}$ of $9.5 \pm 0.6^{\circ}$ and $\delta^{13} \mathrm{C}$ of $-4 \pm 0.8 \%$ and are in equilibrium with other silicate minerals at temperatures of about $600{ }^{\circ} \mathrm{C}$ (van Wyck et al., 1996). The authors interpreted the carbon isotope signature of the fluid as being best modelled as derived from dewatered sediments. The $\mathrm{Sr}$ isotopes, while variable, are more characteristic of a continental crust rather than mantle component. The variability in $\mathrm{Sr}$ isotopes may well reflect metasomatism and/or retrograde metamorphism of the marbles from initial crustal values. If the metasomatic breakdown of minerals in granulites, such as scapolite, contributed to the formation of the eclogite facies marbles and calc-silicates this could account for some of the variability and apparent crustal isotopic signatures. The infiltration of fluids along shear zones can dramatically effect the ${ }^{87} \mathrm{Sr} /{ }^{86} \mathrm{Sr}$ ratios. McCaig et al. (2000) documented infiltration of brine rich fluids rich in $\mathrm{Sr}$ can significantly effect the ${ }^{87} \mathrm{Sr} /{ }^{86} \mathrm{Sr}$ composition of carbonate rocks and accounts 
for much of the variability in the isotopic ratios. Metasomatic skarns in the Grenville have significantly elevated $\mathrm{Sr}$ contents and the partitioning $\mathrm{Sr}$ between scapolite and calcite could potentially significantly modify the $\mathrm{Sr}$ isoptopic system (Shaw et al., 1960).

A metasomatic origin of the marbles and calcsilicates is plausible based on the field relations and is also consistent with the petrological observations. The marbles and calc-silicates occur along or subparallel to Caledonian shear zones. The shear zones served as channel ways for infiltrating hydrous fluids during eclogite facies metamorphism and localized retrogression (Austrheim, 1987; Jamtveit et al., 1990). Some of the surrounding granulites, particularly with anorthositic precusors, contain scapolite. Textural evidence indicates carbonates and $\mathrm{Ca}-\mathrm{Ba}-\mathrm{Sr}$ sulphates forming during breakdown of $\mathrm{SO}_{4}-\mathrm{CO}_{2}$ scapolite in granulites during eclogite facies metamorphism. These observations suggest that the formation of minor carbonates in the eclogites (sensu stricto) from Holsnøy resulted from the breakdown of scapolite in the surrounding granulites. One intriguing possibility is that this breakdown of scapolite in the granulites during high-pressure metamorphism could have contributed to the formation of the localized marbles and calc-silcates along the shear zones. This would imply that the marbles themselves are a product of metasomatic reactions and precipitated from a fluid along shear zones during the high-pressure metamorphism.

\section{Eclogite facies carbonates from the Lindås Nappe: insights into metamorphism of the subducted crust}

Large intact areas of crust on Earth are currently being subducted to depths of $50 \mathrm{~km}$ or more. Continental crust is currently being subducted to great depths beneath the Himalayas, particularly evident in the Pamir region in NW Himalayas (Burtman \& Molnar, 1993), depths at which high-pressure and ultrahigh pressure metamorphism is expected to occur. Exposures of the arrested stages of eclogite metamorphism in the mafic rocks and now the discovery of eclogite facies marbles from Holsnøy from the Lindås Nappe in the Caledonian Orogen of western Norway provides clues to metamorphic processes and possible fluid evolution as the crust is subducted and recycled. The Lindås Nappe anorthosite complex and related rocks represent a slice of lowermost crust that was subducted to high-pressure eclogite facies conditions. However, the timing of high-pressure metamorphism is directly related to the infiltration and access of fluids in the subducted crust (Austrheim \& Envik, 1997; Bingen et al., 2001). The marbles reflect the same high-pressure metamorphic evolution and retain well-preserved eclogitic assemblages. In contrast to some of the mafic eclogites in the area, which equilibrated with evolved saline fluids (Svensen et al., 1999, 2001), fluids in equilibrium with the marbles and calc-silicates appear to be $\mathrm{H}_{2} \mathrm{O}$-rich oxidizing fluids under eclogite facies conditions. The marbles and related calc-silicates may represent the reaction breakdown products of the $\mathrm{SO}_{4}-\mathrm{CO}_{2}$ scapolites in the granulites during highpressure metamorphism. The Sr-rich sulphates and carbonate products could then have been concentrated along Caledonian shear zones in relations to the infiltration of fluids. Further quantification of such a metasomatic origin of the eclogite facies carbonates is the subject on continued investigations.

\section{ACKNOWLEDGEMENTS}

J.F. Molina provided a constructive review of an earlier version of the manuscript and his insights are greatly appreciated. L. Boatner generously provided the $\mathrm{REEPO}_{4}$ standards used in this study which were carefully characterized for use as electron microprobe standards by C. DeWolf. This research was funded by NSF grants 92-095649 and 96-28196 to EJE and 9909697 to TMB. Grants from the Scott Turner Fund, University of Michigan and the Geological Society of America to TMB provided partial support for the field research. The authors are grateful to workers at the University Lausanne for whole rock XRF analyses. B.W.D. Yardley and an anonymous reviewer are thanked for thoughtful and careful reviews. C.E. Manning is thanked for stimulating conversations about fluid composition and for his expertise in aqueous geochemistry. The electron microprobe at the University of Michigan was purchased with NSF grant EAR 82-12764.

\section{REFERENCES}

Andersen, T., Austrheim, H. \& Burke, E. A. J., 1990. Fluid inclusions in granulites and eclogites from the Bergen Arcs, Caledonides of W. Norway. Mineralogical Magazine, 54, 145-158.

Andersen, T. B. \& Jamtveit, B., 1990. Uplift of deep crust during orogenic extensional collapse: a model based on field observations in the Sogn-Sunnfjord region of W. Norway. Tectonics, 9, 1097-1111.

Anovitz, L. M. \& Essene, E. J., 1987. Phase equilibria in the system $\mathrm{CaCO}_{3}-\mathrm{MgCO}_{3}-\mathrm{FeCO}_{3}$. Journal of Petrology, 28, 389-414.

Aranovich, L. \& Newton, R. C., 1996. $\mathrm{H}_{2} \mathrm{O}$ activity in concentrated $\mathrm{NaCl}$ solutions at high pressures and temperatures measured by the brucite-periclase equilibrium. Contributions to Mineralogy and Petrology, 125, 200-212.

Austrheim, H., 1987. Eclogitisation of lower crustal granulites by fluid migration through shear zones. Earth and Planetary Science Letters, 81, 221-232.

Austrheim, H., 1990. Fluid induced processes in the lower crust as evidenced by Caledonian eclogitization of Precambrian granulites, Bergen Arcs, western Norway. PhD Thesis, Universitetet i Oslo, Oslo.

Austrheim, H. \& Boundy, T. M., 1994. Pseudotachylytes generated during seismic faulting and eclogitization of the deep crust. Science, 265, 82-83.

Austrheim, H. \& Envik, A., 1997. Fluid transport, deformation and metamorphism at depth in a collision zone. In: Fluid Flow and Transport in Rocks: Mechanisms and Effects (eds Jamtveit, B. \& Yardley, B. W. D.), pp. 123-137. Chapman \& Hall, London. 
Austrheim, H., Erambert, M. \& Engvik, A. K., 1997. Processing of crust in the root of the Caledonian continental collision zone: the role of eclogitization. Tectonophysics, 273, 129-153.

Austrheim, H. \& Griffin, W. L., 1985. Shear deformation and eclogite formation within the granulite-facies anorthosites of the Bergen Arcs, western Norway. Chemical Geology, 50, 267-281.

Baldasari, A. \& Speer, J. A., 1979. Witherite composition, physical properties, and genesis. American Mineralogist, 64, 742-747.

Bingen, B., Austrheim, H. \& Whitehouse, M., 2001. Ilmenite as a source for zirconium during high-grade metamorphism? Textural evidence from the caledonides of Western Norway and implications for zircon geochronology. Journal of Petrology, 42, 355-375.

Boundy, T. M., 1995. Metamorphic, geochronologic and tectonic evolution of deep crustal rocks within the Caledonian Orogen, western Norway, PhD Thesis, University of Michigan, Ann Arbor.

Boundy, T. M., Essene, E. J. \& Mezger, K., 1997a. Temporal and tectonic evolution of the granulite-eclogite association from the Bergen Arcs, western Norway. Lithos, 39, 159-178.

Boundy, T. M., Fountain, D. M. \& Austrheim, H., 1992. Structural development and petrofabrics of eclogite facies shear zones in the granulite facies complex, Bergen Arcs, western Norway: implications for deep crustal deformational processes. Journal of Metamorphic Geology, 10, 127-146.

Boundy, T. M., Hall, C. M., Li, G., Essene, E. J. \& Halliday, A. N., 1997b. Fine-scale isotopic heterogeneities and fluids in the deep crust: ${ }^{40} \mathrm{Ar} /{ }^{39} \mathrm{Ar}$ laser ablation and TEM study of muscovites from a granulite-eclogite transition zone. Earth and Planetary Science Letters, 148, 232-242.

Burtman, V. S. \& Molnar, P., 1993. Geological and geophysical evidence for deep subduction of continental crust beneath Pamir. Geological Society of America Special Paper, 281.

Caby, R., 1994. Precambrian coesite from northern Mali: first record and implications for plate tectonics in the transSaharan segment of the Pan-African belt. European Journal of Mineralogy, 6, 235-244.

Carlson, W. D., 1980. The calcite - aragonite equilibrium: effects of $\mathrm{Sr}$ substitution and anion orientation disorder. American Mineralogist, 65, 1252-1262.

Casey, W. H., Chai, L., Navrotsky, A. \& Rock, P. A., 1996. Thermochemistry of mixing strontianite $\left[\mathrm{SrCO}_{3}\right.$ (s)] and aragonite $\left[\mathrm{CaCO}_{3}\right.$ (s)] to form $\mathrm{Ca}_{\mathrm{x}} \mathrm{Sr}_{1-\mathrm{x}} \mathrm{CO}_{3}$ (s) solid solutions. Geochimica et Cosmochimica Acta, 60, 933-940.

Castelli, D., 1991. Eclogitic metamorphism in carbonate rocks: the example of impure marbles from the Sesia-Lanzo Zone, Italian Western Alps. Journal of Metamorphic Geology, 9, 61-77.

Dasgupta, S., Chakraborti, S., Fukuoku, M., Sengupta, P. \& Roy, S., 1990. Ca-Ba-Sr carbonates from metamorphosed manganese deposits of the Sausar group, India, and their petrological significance. Mineralogical Magazine, 54, 511513.

Deer, W. A., Howie, R. A. \& Zussman, J., 1962. Rock Forming Minerals, Vol. 5: Non-Silicates. Longman Press, New York.

DeWolf, C., 1993. Investigation of monazite and garnet geochemistry and applications to the Archean gneiss terrane of the Wind River Range, Wyoming. PhD Thesis, University of Michigan, Ann Arbor.

Donohue, C. L. \& Essene, E. J., 2000. An oxygen barometer with the assemblage garnet-epidote. Earth and Planetary Science Letters, 181, 459-472.

Edwards, R. L. \& Essene, E. J., 1988. Pressure, temperature and $\mathrm{C}-\mathrm{O}-\mathrm{H}$ fluid fugacities across the amphibolite-granulite transition, NW Adirondack Mountains, NY. Journal of Petrology, 29, 39-72.

Essene, E. J., 1983. Solid solutions and solvi among metamorphic carbonates with applications to geologic thermometry. In. Carbonates: Mineralogy and Chemistry, Reviews in Mineralogy, 10. (ed. Reeder, R. J.), pp. 105-206. Mineralogical Society of America, Washington DC.
Franz, G. \& Spear, F. S., 1983. High-pressure metamorphism of siliceous dolomites from the central Tauern window. American Journal of Science, 283A, 396-413.

Getty, S. R. \& Selverstone, J., 1994. Stable isotopic and trace element evidence for restricted fluid migration in $2 \mathrm{GPa}$ eclogites. Journal of Metamorphic Geology, 12, 747-766.

Holland, T. J. B., 1979. High water activities in the generation of high pressure kyanite eclogites of the Tauern Window, Austria. Journal of Geology, 87, 1-27.

Holland, T. J. B., 1980. The reaction albite $=$ jadeite + quartz determined experimentally in the range 600-1200 ${ }^{\circ} \mathrm{C}$. American Mineralogist, 65, 129-134.

Holland, T. J. B. \& Powell, R., 1998. An internally consistent thermodynamic dataset for phases of petrological interest. Journal of Metamorphic Geology, 16, 309-343.

Jamtveit, B., Bucher-Nurminen, K. \& Austrheim, H., 1990. Fluid controlled eclogitization of granulites in deep crustal shear zones, Bergen Arcs, western Norway. Contributions to Mineralogy and Petrology, 104, 184-193.

Kato, T., Enami, M. \& Zhai, M., 1997. Ultra-high-pressure (UHP) marble and eclogite in the Su-Lu UHP terrane, eastern China. Journal of Metamorphic Geology, 15, 169-182.

Klaper, E. M., 1991. Eclogite shear zones in a granulite-facies anorthosite complex: field relationships and emplacement scenario - an example from the Bergen Arcs, Western Norway. Schweizerische Mineralogische und Petrographische. Mitteilungen, 71, 231-241.

Klemd, R., Matthes, S. \& Schuessler, U., 1994. Reaction textures and fluid behaviour in very high-pressure calc-silicate rocks of the Muenchberg Gneiss Complex, Bavaria, Germany. Journal of Metamorphic Geology, 12, 735-745.

Krogh Ravna, E., 2000. The garnet-clinopyroxene $\mathrm{Fe}^{2+}-\mathrm{Mg}$ geothermometer: an updated calibration. Journal of Metamorphic Geology, 18, 211-219.

Krogh, E. J., 1988. The garnet-clinopyroxene geothermometera reinterpretation of existing experimental data. Contributions to Mineralogy and Petrology, 99, 44-48.

Liu, L.-G. \& Bassett, W. A., 1986. Elements, Oxides, and Silicates. Oxford University Press, New York.

Manning, C. E., 1994. The solubility of quartz in $\mathrm{H}_{2} \mathrm{O}$ in the lower crust and upper mantle. Geochimica et Cosmochimica Acta, 58, 4831-4839.

McCaig, A. M., Tritlla, J. \& Banks, D. A., 2000. Fluid mixing and recycling during Pyrenean thrusting: evidence from fluid inclusion halogen ratios. Geochimica et Cosmochimica Acta, 64, 3395-3412.

Moecher, D. P. \& Essene, E. J., 1990. Phase equilibria for calcic scapolite, and implications of variable Al-Si disorder on $P-T, T-X_{\mathrm{CO} 2}$, and $a-X$ relations. Journal of Petrology, 31, 997-1024.

Molina, J. F. \& Poli, S., 2000. Carbonate stability and fluid composition in subducted oceanic crust: experimental study on $\mathrm{CO}_{2}-\mathrm{H}_{2} \mathrm{O}$-bearing basalts. Earth and Planetary Science Letters, 176, 295-310.

Munoz, J. L., 1992. Calculation of $\mathrm{HF}$ and $\mathrm{HCl}$ fugacities from biotite compositions; revised equations. GSA Abstracts with Programs, 24, 221.

Munoz, J. L. \& Swenson, A., 1981. Chloride-hydroxyl exchange in biotite and estimation of relative $\mathrm{HCl}-\mathrm{HF}$ activities in hydrothermal fluids. Economic Geology, 76, 2212-2221.

Peacock, S. M., 1992. The importance of blueschist $->$ eclogite dehydration reactions in subducting oceanic crust. Geological Society of America Bulletin, 105, 684-694.

Pisarskii, B. I., Konev, A. A., Levi, K. G. \& Delvaux, D., 1998. Alkaline carbon dioxide hydrotherms and strontium-containing travertines in the Songwe River Valley (Tanzania). Geologiia Y Geofizika, 39, 934-941.

Platt, R. G. \& Woolley, A. R., 1990. The carbonatites and fenites of Chipman Lake, Ontario. Canadian Mineralogist, 28, 241-250.

Prieto, M., Fernandez-Gonzalez, A., Putnis, A. \& FernandezDiaz, L., 1997. Nucleation, growth, and zoning phenomena in crystallizing $(\mathrm{Ba}, \mathrm{Sr}) \mathrm{CO}_{3}, \mathrm{Ba}\left(\mathrm{SO}_{4}, \mathrm{CrO}_{4}\right),(\mathrm{Ba}, \mathrm{Sr}) \mathrm{SO}_{4}$ 
and (Cd,Ca) $\mathrm{CO}_{3}$. Geochimica et Cosmochimica Acta, 61, 3383-3397.

Roberts, D. \& Gee, D. G., 1985. An introduction to the structure of the Scandinavian Caledonides. In: The Caledonide Orogen Scandinavia and Related Areas (eds Gee, D. G. \& Sturt, B. A.), pp. 55-68. John Wiley \& Sons, London.

Robie, R. A. \& Hemingway, B. S., 1995. Thermodynamic Properties of Minerals and Related Substances at $298.15 \mathrm{~K}$ and 1 Bar $\left(10^{5}\right.$ Pascals) Pressure and at Higher Temperatures. Bulletin 2131 U US Geological Survey, Reston.

Rockow, K. M., Haskin, L. A., Jolliff, B. L. \& Fountain, D. M., 1997. Constraints on element mobility associated with the conversion of granulite to eclogite along fractures in an anorthosite complex on Holsnøy, Norway. Journal of Metamorphic Geology, 15, 401-418.

Schertl, H.-P. \& Okay, A. L., 1994. A coesite inclusion in dolomite in Dabie Shan, China: petrological and rheological significance. European Journal of Mineralogy, 6, 995-1000.

Scott, J. D. \& Peatfield, G. R., 1986. Mannardite $\left[\mathrm{BaH}_{2} \mathrm{O}\right]$ $\left(\mathrm{Ti}_{6} \mathrm{~V}_{2}^{3+} \mathrm{O}_{16}\right)$, a new mineral species and new data on redledgeite. The Canadian Mineralogist, 24, 55-66.

Selverstone, J., Franz, G., Thomas, S. \& Getty, S. R., 1992. Fluid variability in $2 \mathrm{GPa}$ eclogites as a indicator of fluid behaviour during subduction. Contributions to Mineralogy and Petrology, 112, 341-357.

Shaw, D. M., Moxham, R. L. M., Filby, R. H. \& Lapkowsky, W. W., 1960. The Petrology and Geochemistry of Grenville Skarns. Part II: Geochemistry. Canadian Mineralogist, 7, 420-442.

Shmulovitch \& Graham, C. M., 1999. An experimental study of phase equilibria in the system $\mathrm{H}_{2} \mathrm{O}-\mathrm{CO}_{2}-\mathrm{NaCl}$ at $800{ }^{\circ} \mathrm{C}$ and 9 kbar. Contributions to Mineralogy and Petrology, 136, $247-257$.

Speer, J. A., 1983. Crystal chemistry and phase relations of orthorhombic carbonates. In: Carbonates: Mineralogy and Chemistry, Reviews in Mineralogy 11 (ed. Reeder, R. J.). pp. 145-190. Mineralogical Society of America, Washington DC.
Svensen, H., Jamtveit, B., Banks, D. A. \& Austrheim, H., 2001. Halogen contents of eclogite-facies fluid inclusions and minerals: Caledonides, western Norway. Journal of Metamorphic Geology, 19, 165-178.

Svensen, H., Jamtveit, B., Yardley, B., Engvik, A., Austrheim, H. \& Broman, C., 1999. Lead and bromine enrichment in eclogite facies fluids: extreme fractionation during lowercrustal hydration. Geology, 27, 467-470.

Vorob'yev, Y. I., Konev, A. A., Malyshonok, Y. V. \& Piskunova, L. F., 1999. Subsolidus phase transitions of the carbonate component of strontium-barium carbonatite. Doklady Akademii Nauk SSSR, 304, 1449-1452.

Wang, X. \& Liou, J. G., 1991. Regional ultra-high-pressure coesite-bearing eclogitic terrane in central China: evidence from country rocks, gneiss, marble, and metapelite. Geology, 19, 933-936.

Wang, X. \& Liou, J. G., 1993. Ultra-high-pressure metamorphism of carbonate rocks in the Dabie Mountains, central China. Journal of Metamorphic Geology, 11, 575-588.

Woolley, A. R. \& Kempe, D.R., 1989. Carbonatites: nomenclature, average chemical compositions, and element distribution. In: Carbonatites. Genesis and Evolution. (ed. Bell, K.), pp. 1-14. Unwyn Hyman, London.

van Wyck, N., Valley, J. W. \& Austrheim, H., 1996. Oxygen and carbon isotope constraints on the development of eclogites, Holsnøy, Norway. Lithos, 38, 129-145.

Yardley, B. W. D., 1997. The evolution of fluids though the metamorphic cycle. In: Fluid Flow and Transport in Rocks: Mechanisms and Effects (eds Jamtveit, B. \& Yardley, B. W. D.), pp. 99-122. Chapman \& Hall, London.

Zhang, R. Y. \& Liou, J. G., 1996. Coesite inclusions in dolomite from eclogite in the southern Dabie Mountains, China: the significance of carbonate minerals in UHPM rocks. American Mineralogist, 11, 575-588.

Received 23 February 2001; revision accepted 23 March 2002. 